PHYSICAL REVIEW D 81, 084054 (2010)

\title{
Final spins from the merger of precessing binary black holes
}

\author{
Michael Kesden* \\ California Institute of Technology, MC 350-17, 1216 E. California Boulevard, Pasadena, California 91125, USA \\ Ulrich Sperhake ${ }^{\dagger}$ and Emanuele Berti \\ Department of Physics and Astronomy, The University of Mississippi, University, Mississippi 38677-1848, USA, \\ and California Institute of Technology, MC 350-17, 1216 E. California Boulevard, Pasadena, California 91125, USA
}

(Received 25 February 2010; published 29 April 2010)

\begin{abstract}
The inspiral of binary black holes is governed by gravitational radiation reaction at binary separations $r \lesssim 1000 M$, yet it is too computationally expensive to begin numerical-relativity simulations with initial separations $r \gtrsim 10 M$. Fortunately, binary evolution between these separations is well described by postNewtonian equations of motion. We examine how this post-Newtonian evolution affects the distribution of spin orientations at separations $r \simeq 10 M$ where numerical-relativity simulations typically begin. Although isotropic spin distributions at $r \simeq 1000 M$ remain isotropic at $r \simeq 10 M$, distributions that are initially partially aligned with the orbital angular momentum can be significantly distorted during the postNewtonian inspiral. Spin precession tends to align (antialign) the binary black hole spins with each other if the spin of the more massive black hole is initially partially aligned (antialigned) with the orbital angular momentum, thus increasing (decreasing) the average final spin. Spin precession is stronger for comparable-mass binaries and could produce significant spin alignment before merger for both supermassive and stellar-mass black hole binaries. We also point out that precession induces an intrinsic accuracy limitation ( $\lesssim 0.03$ in the dimensionless spin magnitude, $\lesssim 20^{\circ}$ in the direction) in predicting the final spin resulting from the merger of widely separated binaries.
\end{abstract}

DOI: 10.1103/PhysRevD.81.084054

\section{INTRODUCTION}

The existence of black holes is a fundamental prediction of general relativity. Isolated individual black holes are stationary solutions to Einstein's equations, but binary black holes (BBHs) can inspiral and eventually merge. BBH mergers offer a unique opportunity to test general relativity in the strong-field limit and as such are a primary science target for current and future gravitational-wave (GW) observatories like LIGO, VIRGO, LISA, and the Einstein telescope. BBH mergers are also important for cosmology, as they can serve as standard candles to help determine the geometry and hence energy content of the universe [1,2]. Astrophysical BBHs are found on at least two very different mass scales. Compact objects believed to be stellar-mass black holes have been observed in binary systems with more luminous companions. These black holes are the remnants of massive main-sequence stars, and binary systems with two such stars may ultimately evolve into BBHs. On larger scales, supermassive black holes (SBHs) with masses $10^{6} \lesssim M / M_{\odot} \lesssim 10^{9}$ reside in the centers of most galaxies. They can be observed through their dynamical influence on surrounding gas and stars, as well as when accreting as active galactic nuclei. SBHs will form binaries as well, following the merger of two galaxies that each host an SBH at their center.

\footnotetext{
*kesden@tapir.caltech.edu

†sperhake@tapir.caltech.edu

berti@phy.olemiss.edu
}

PACS numbers: 04.25.dg, 04.25.Nx, 04.30.Tv, 04.70.-s

In order to merge, BBHs must find a way to shed their orbital angular momentum. At large separations, binary SBHs will be escorted inwards by dynamical friction between their host galaxies [3]. The BBHs become gravitationally bound when the sum of their masses $M \equiv m_{1}+m_{2}$ exceeds the mass of gas and stars enclosed by their orbit. The binary hardens further by scattering stars on "loss-cone" orbits that pass within a critical radius [4], though this scattering may stall at separations $r \simeq$ $0.01-1$ pc unless these orbits are refilled by stellar diffusion [5]. Unlike stars, gas can cool to form a circumbinary disk about the BBHs. A circumbinary disk of mass $M_{d}$ and radius $r_{d}$ will exert a tidal torque

$$
T_{d} \sim \frac{q^{2} M_{d} M}{r}\left(\frac{r}{r_{d}-r}\right)^{3}
$$

on the binary in the limit that the $\mathrm{BBH}$ mass ratio $q \equiv$ $m_{2} / m_{1} \leq 1$ is small and $\left|r_{d}-r\right| \ll r$ [6-8]. Throughout this paper we use relativists' units in which Newton's constant $G$ and the speed of light $c$ are unity. At a sufficiently small separation $r_{\mathrm{GW}}$, the magnitude of this tidal torque will fall below that of the radiation-reaction torque [9]

$$
T_{\mathrm{GW}}=\frac{32 \eta^{2} M^{9 / 2}}{5 r^{7 / 2}}
$$

where $\eta \equiv m_{1} m_{2} / M^{2}$ is the symmetric mass ratio. Once $T_{\mathrm{GW}}>T_{d}$, the inspiral of the $\mathrm{BBH}$ is dominated by radiation reaction. The precise value of $r_{\mathrm{GW}}$ depends on the 
properties of the circumbinary disk, but an order-ofmagnitude estimate is given by [3]

$$
\begin{aligned}
r_{\mathrm{GW}} & =\left(5 \times 10^{16} \mathrm{~cm}\right) q^{1 / 4} M_{8}^{3 / 4}\left[\frac{\min \left(t_{h}, t_{\mathrm{gas}}\right)}{10^{8} \mathrm{yr}}\right]^{1 / 4} \\
& =(3000 M)\left(\frac{q}{M_{8}}\right)^{1 / 4}\left[\frac{\min \left(t_{h}, t_{\mathrm{gas}}\right)}{10^{8} \mathrm{yr}}\right]^{1 / 4},
\end{aligned}
$$

where $M_{8}$ is the mass of the larger black hole in units of $10^{8} M_{\odot}, t_{h}$ is the dynamical friction time scale for a hard binary, and $t_{\mathrm{gas}}$ is the evolution time scale from gaseous tidal torques.

General relativity completely determines the inspiral of $\mathrm{BBH}$ systems from separations less than $r_{\mathrm{GW}}$. These systems are fully specified by 7 parameters: the mass ratio $q$ and the 3 components of each dimensionless spin $\boldsymbol{\chi}_{1,2} \equiv$ $\mathbf{S}_{1,2} / m_{1,2}^{2}$. To a good approximation the individual masses and spin magnitudes $\chi_{1,2} \equiv\left|\chi_{1,2}\right|$ remain constant during the inspiral, so only the precession of the two spin directions needs to be calculated. At an initial separation $r_{i}=$ $1000 M \sim r_{\mathrm{GW}}$, the binary's orbital speed $v / c \ll 1$ and the spin-precession equations can therefore be expanded in this small post-Newtonian (PN) parameter. The PN expansion remains valid until the BBHs reach a final separation $r_{f}=10 M$, after which their evolution can be described only by fully nonlinear numerical relativity (for more precise assessments of the validity of the PN expansion for binaries with precessing spins, see e.g. $[10,11])$. Numerical relativists can simulate BBH mergers from separations $r_{\mathrm{NR}} \simeq r_{f}$ [12-14], but these simulations are too computationally expensive to begin when the binaries are much more widely separated. The GWs produced in the merger and the mass, spin, and recoil velocity of the final black hole depend sensitively on the orientation of the $\mathrm{BBH}$ spins at $r_{\mathrm{NR}}$, so it is important to determine what $\mathrm{BBH}$ spin orientations are expected at $r_{i}$ and whether these orientations are modified by the PN evolution between $r_{i}$ and $r_{f}$.

The answer to the first of these questions comes from astrophysics, not general relativity. At very large separations, the two black holes are unaffected by each other and one would therefore expect an isotropic distribution of spin directions. However, an isotropic distribution of spins at $r_{f}$ would imply that most mergers would result in a gravitational recoil of $\sim 1000 \mathrm{~km} / \mathrm{s}$ for the final black hole [1517]. Recoils this large would eject SBHs from all but the most massive host galaxies [18], in seeming contradiction to the observed tight correlations between SBHs and their hosts [19-21]. This problem can be avoided if LenseThirring precession and viscous torques align the spins of the BBHs with the accretion disk responsible for their inwards migration [22-24]. The efficiency of this alignment depends on the properties of the accretion disk, but $N$-body simulations using smoothed-particle hydrodynam- ics suggest that the residual misalignment of the $\mathrm{BBH}$ spins with their accretion disk at $r_{i}$ could typically be $\sim 10^{\circ}\left(30^{\circ}\right)$ for cold (hot) accretion disks [17].

The second question (does the distribution of spin directions change as the BBHs inspiral from $r_{i}$ to $r_{f}$ ?) can be answered by evolving this distribution over this interval using the PN spin-precession equations. We will describe these PN equations and our numerical solutions to them in Sec. II. The precession of a given spin configuration in the PN regime can be understood in terms of the proximity of that configuration to the nearest spin-orbit resonance. Schnittman [25] identified a set of equilibrium spin configurations in which both black hole spins and the orbital angular momentum lie in a plane, along with the total angular momentum $\mathbf{J}=\mathbf{L}+m_{1}^{2} \boldsymbol{\chi}_{1}+m_{2}^{2} \boldsymbol{\chi}_{2}$. In the absence of radiation reaction, $\mathbf{J}$ is conserved. For these equilibrium configurations, the spins and orbital angular momentum remain coplanar and precess jointly about $\mathbf{J}$ with the angles $\theta_{1,2}$ between $\mathbf{L}$ and $\boldsymbol{\chi}_{1,2}$ remaining fixed. The equilibrium configurations can thus be understood as spin-orbit resonances since the precession frequencies of $\mathbf{L}$ and $\boldsymbol{\chi}_{1,2}$ about $\mathbf{J}$ are all the same. Once radiation reaction is added, the spins and orbital angular momentum remain coplanar as the BBHs inspiral, although $\theta_{1}$ and $\theta_{2}$ slowly change on the inspiral time scale. Not only do resonant configurations remain resonant, but configurations near resonance can be captured into resonance during the inspiral. The resonances are thus very important for understanding the evolution of generic BBH systems, although the resonances themselves occupy only a small portion of the seven-dimensional parameter space characterizing generic mergers. We shall review these spin-orbit resonances in more detail in Sec. III.

Bogdanović et al. [23] briefly considered whether spinorbit resonances could effectively align SBH spins with the orbital angular momentum following the merger of gaspoor galaxies. They found that for a mass ratio $q=9 / 11$ and maximal spins $\chi_{1}=\chi_{2}=1$, an isotropic distribution of spins at $r_{i}=1000 \mathrm{M}$ remains isotropically distributed when evolved to $r_{f}=10 \mathrm{M}$. They therefore concluded that an alternative mechanism, such as the accretion torques considered later in their paper, is needed to align the BBH spins with $\mathbf{L}$. This conclusion is supported by a much larger set of PN inspirals presented by Herrmann et al. [26] who found that for equal-mass BBHs, an isotropic distribution of spins at $40 M$ yields a flat distribution in $\cos \theta_{12}$ at $7.4 M$. Here and in this paper $\theta_{12}$ is the angle between the two spins $\boldsymbol{\chi}_{1}$ and $\boldsymbol{\chi}_{2}$. In the final plot of their paper, Herrmann et al. [26] revealed their discovery of an anticorrelation between the initial and final values of $\cos \theta_{12}$ for $q=2 / 3 \mathrm{BBHs}$ with equal dimensionless spins $\chi_{1}=\chi_{2}=0.05$. Investigation of this anticorrelation was left to future work. Lousto et al. [27] also found indications that an initially isotropic distribution of spins can become nonisotropic during the PN stage of the inspiral. For a 
range of mass ratios $1 / 16 \leq q \leq 1$ and equal spins $\chi_{1}=$ $\chi_{2}=(0.485,0.686,0.97)$, they found that an isotropic spin distribution at $50 \mathrm{M}$ develops a slight but statistically significant tendency toward antialignment with the orbital angular momentum $\mathbf{L}$. This amplitude of antialignment scales linearly in the BBH spin magnitudes and appears to decrease as $q \rightarrow 0$.

We perform our own study of PN spin evolution from $r_{i}$ to $r_{f}$ for several reasons. BBHs get locked into spin-orbit resonances at a separation

$$
r_{\text {lock }} \propto\left(\frac{\chi_{1} \cos \theta_{1}-q^{2} \chi_{2} \cos \theta_{2}}{1-q^{2}}\right)^{2} M,
$$

which can become large in the equal-mass $(q \rightarrow 1)$ limit [25]. This limit is important, as the largest recoil velocities occur for nearly equal-mass mergers. Numerical integration of the PN equations has shown that for a mass ratio $q=9 / 11$, spin-orbit resonances affect spin orientations at separations $r \simeq 1000 M$. This is a much larger separation than was considered in previous studies [26,27] of spin alignment, which may therefore have failed to capture the full magnitude of the effect. These studies also focused on whether an initially isotropic distribution of spins becomes anisotropic just prior to merger. However, as discussed above, tidal torques from a circumbinary disk partially align spins with the orbital angular momentum at separations $r \gg r_{\mathrm{GW}}$ before relativistic effects become important. As we will show in Sec. IV, such partially aligned distributions can be strongly affected by spin-orbit resonances despite the fact that isotropic distributions remain nearly isotropic. We will consider how spin precession affects the final spin magnitudes and directions in Sec. V. The evolution of the distribution of BBH spin directions between $r_{i}$ and $r_{f}$ changes the distribution of final spin magnitudes and directions from what it would have been in the absence of precession. In addition, spin precession introduces a fundamental uncertainty in predicting the final spin of a given BBH system. At large separations, a small uncertainty in the separation leads to an uncertainty in the predicted time until merger that exceeds the precession time. In this case, one cannot predict at what phase of the spin precession the merger will occur and thus the resulting final spin. We will explore this uncertainty in Sec. VI. A brief discussion of the chief findings of this paper is given in Sec. VII.

\section{POST-NEWTONIAN EVOLUTION}

We evolve spinning BBH systems along a sequence of quasicircular orbits according to the PN equations of motion for precessing binaries first derived by Kidder [28], and later used by Buonanno, Chen, and Vallisneri to build matched-filtering template families for GW detection [29]. The adiabatic evolution of the binary's orbital frequency is described including terms up to 3.5PN order, and spin effects are included up to $2 \mathrm{PN}$ order. These evolution equations were chosen for consistency with previous work, in particular, with the study by Barausse and Rezzolla [30] of the final spin resulting from the coalescence of BBHs and with the statistical investigation of spinning BBH evolutions using Graphics Processing Units by Herrmann et al. [26]. Lousto et al. [27] evolved a large sample of spinning BBH systems using a nonresummed, PN expanded Hamiltonian. The convergence properties of non-resummed Hamiltonians for spinning BBH systems are somewhat problematic (see e.g. Fig. 1 of Ref. [10]), and it will be interesting to repeat these statistical investigations of precessing BBH systems using the effective-one-body resummations of the PN Hamiltonian recently proposed by Barausse et al. [31,32].

In our simulations, the spins evolve according to

$$
\begin{aligned}
& \dot{\mathbf{S}}_{1}=\overline{\mathbf{\Omega}}_{1} \times \mathbf{S}_{1}, \\
& \dot{\mathbf{S}}_{2}=\overline{\mathbf{\Omega}}_{2} \times \mathbf{S}_{2},
\end{aligned}
$$

where

$\overline{\mathbf{\Omega}}_{1}=\frac{1}{2 r^{3}}\left[\left(4+3 q-\frac{3\left(\mathbf{S}_{2}+q \mathbf{S}_{1}\right) \cdot \mathbf{L}_{N}}{L_{N}^{2}}\right) \mathbf{L}_{N}+\mathbf{S}_{2}\right]$,

$\overline{\mathbf{\Omega}}_{2}=\frac{1}{2 r^{3}}\left[\left(4+\frac{3}{q}-\frac{3\left(\mathbf{S}_{1}+q^{-1} \mathbf{S}_{2}\right) \cdot \mathbf{L}_{N}}{L_{N}^{2}}\right) \mathbf{L}_{N}+\mathbf{S}_{1}\right]$

are the spin-precession frequencies averaged over a circular orbit, including the quadrupole-monopole interaction [33],

$$
\mathbf{L}_{N}=\eta M \mathbf{r} \times \mathbf{v}=\frac{\eta M^{2}}{(M \omega)^{1 / 3}} \hat{\mathbf{L}}_{N}
$$

is the Newtonian orbital angular momentum, and

$$
\omega=\left(\frac{M}{r^{3}}\right)^{1 / 2}
$$

is the orbital frequency. In the absence of gravitational radiation, $\mathbf{J}$ and $\left|\mathbf{L}_{N}\right|$ are constant, implying that the direction of the orbital angular momentum evolves according to

$$
\dot{\hat{\mathbf{L}}}_{N}=-\frac{(M \omega)^{1 / 3}}{\eta M^{2}} \frac{d \mathbf{S}}{d t},
$$

where $\mathbf{S}=\mathbf{S}_{1}+\mathbf{S}_{2}$. Once radiation reaction is included, the orbital frequency slowly evolves as 


$$
\begin{aligned}
\dot{\omega}= & \omega^{2} \frac{96}{5} \eta(M \omega)^{5 / 3}\left\{1-\frac{743+924 \eta}{336}(M \omega)^{2 / 3}+\left[\left(\frac{19}{3} \eta-\frac{113}{12}\right) \boldsymbol{\chi}_{s} \cdot \hat{\mathbf{L}}_{N}-\frac{113 \delta}{12} \boldsymbol{\chi}_{a} \cdot \hat{\mathbf{L}}_{N}+4 \pi\right](M \omega)\right. \\
& +\left\{\left(\frac{34103}{18144}+\frac{13661}{2016} \eta+\frac{59}{18} \eta^{2}\right)-\frac{\eta \chi_{1} \chi_{2}}{48}\left(247 \hat{\mathbf{S}}_{1} \cdot \hat{\mathbf{S}}_{2}-721\left(\hat{\mathbf{L}}_{N} \cdot \hat{\mathbf{S}}_{1}\right)\left(\hat{\mathbf{L}}_{N} \cdot \hat{\mathbf{S}}_{2}\right)\right)\right. \\
& \left.+\sum_{i=1}^{2} \frac{\left(m_{i} \chi_{i}\right)^{2}}{M^{2}}\left[\frac{5}{2}\left(3\left(\hat{\mathbf{L}}_{N} \cdot \hat{\mathbf{S}}_{i}\right)^{2}-1\right)+\frac{1}{96}\left(7-\left(\hat{\mathbf{L}}_{N} \cdot \hat{\mathbf{S}}_{i}\right)^{2}\right)\right]\right\}(M \omega)^{4 / 3}-\frac{4159+15876 \eta}{672} \pi(M \omega)^{5 / 3} \\
& +\left[\left(\frac{16447322263}{139708800}-\frac{1712 \gamma_{E}}{105}+\frac{16 \pi^{2}}{3}\right)+\left(-\frac{273811877}{1088640}+\frac{451 \pi^{2}}{48}-\frac{88}{3} \hat{\theta} \eta\right) \eta+\frac{541}{896} \eta^{2}-\frac{5605}{2592} \eta^{3}\right. \\
& \left.\left.-\frac{856}{105} \log \left[16(M \omega)^{2 / 3}\right]\right](M \omega)^{2}+\left(-\frac{4415}{4032}+\frac{358675}{6048} \eta+\frac{91495}{1512} \eta^{2}\right) \pi(M \omega)^{7 / 3}\right\},
\end{aligned}
$$

where $\gamma_{E} \simeq 0.577$ is Euler's constant, $\hat{\theta} \equiv 1039 / 4620$, and we have defined

$$
\begin{aligned}
& \boldsymbol{\chi}_{s} \equiv \frac{1}{2}\left(\boldsymbol{\chi}_{1}+\boldsymbol{\chi}_{2}\right), \\
& \boldsymbol{\chi}_{a} \equiv \frac{1}{2}\left(\boldsymbol{\chi}_{1}-\boldsymbol{\chi}_{2}\right) .
\end{aligned}
$$

The two terms in square parentheses on the third line of Eq. (2.6) are due to the quadrupole-monopole interaction [34] and to the spin-spin self-interaction [35], respectively, and they were neglected in the statistical study of Ref. [26]. Their sum agrees with Eq. (5.17) of Ref. [36]. Additional simulations performed without these terms show our results to be insensitive to their inclusion in the evolution of the orbital frequency.

The numerical integration of this system of ordinary differential equations is performed using the adaptive stepsize integrator STEPPERDOPR5 [37]. The evolution of any given $\mathrm{BBH}$ system is specified by the following parameters: the initial orbital frequency $\omega_{i}$, the binary's mass ratio $q \equiv m_{2} / m_{1}$, the dimensionless magnitude of each spin $\chi_{i}$, and the relative orientation $\left(\theta_{i}, \phi_{i}\right)$ of each spin with respect to the orbital angular momentum at time $t=0$ $(i=1,2)$. To monitor the variables along the whole evolution, we output all quantities using a constant logarithmic spacing in the orbital frequency at low frequencies, and the stepsize as used in the integrator at high frequencies. Typically this results in a total of about 64000 points in the range $M \omega \in\left[M \omega_{i}, M \omega_{f}\right]$, where $M \omega_{i}=3.16 \times$ $10^{-5}$ and $M \omega_{f}=0.1$. Numerical experimentation indicates that a tolerance parameter ATOL $=2 \times 10^{-8}$ in the adaptive stepsize integrator is sufficient for a pointwise accuracy of order $1 \%$ or better in the final quantities. Therefore the error induced by the numerical integrations of the PN equations of motion is subdominant with respect to the errors induced by precessional effects and by fits of the numerical simulations, which will be one of the main topics of this paper.

\section{SPIN-ORBIT RESONANCES}

In this section, we review the equilibrium configurations of BBH spins first presented in Schnittman [25] for which the Newtonian orbital angular momentum $\mathbf{L}_{N}$ and individ- ual spins $\mathbf{S}_{1,2}$ all precess at the same resonant frequency. As discussed briefly in the Introduction, at a given binary separation $r$ fully general quasicircular BBHs are described by 7 parameters: the mass ratio $q$ and the 3 components of each black hole spin. In spherical coordinates with $\mathbf{L}_{N}$ defining the $z$ axis, each spin is given by its magnitude $S_{i}=m_{i}^{2} \chi_{i}$ and direction $\left(\theta_{i}, \phi_{i}\right)(i=1,2)$. In the PN limit for which this analysis is valid, a clear hierarchy

$$
t_{\text {orb }} \ll t_{p} \ll t_{\mathrm{GW}}
$$

exists between the orbital time $t_{\text {orb }} \propto r^{3 / 2}$, the precession time $t_{p} \sim \Omega_{1,2}^{-1} \propto r^{5 / 2}$, and the radiation time $t_{\mathrm{GW}} \sim$ $\dot{E}_{\mathrm{GW}} / E \propto r^{4}$. This hierarchy implies that the BBH spins will precess many times before merger, leaving only their relative angular separation $\Delta \phi \equiv \phi_{2}-\phi_{1}$ in the orbital plane well defined. This reduces the BBH parameter space to six dimensions. Since the mass ratio and individual spin magnitudes are preserved during the inspiral, a given $\mathrm{BBH}$ evolves through the three-dimensional parameter space $\left(\theta_{1}, \theta_{2}, \Delta \phi\right)$ on the precession time scale $t_{p}$. This evolution is governed by the spin-precession Eqs. (2.1).

Schnittman [25] discovered a one-parameter family of equilibrium solutions to these equations for which $\left(\theta_{1}, \theta_{2}, \Delta \phi\right)$ remain fixed on the precession time scale $t_{p}$. These solutions have $\Delta \phi=0^{\circ}$ or $180^{\circ}$, implying that $\mathbf{L}_{N}$, $\mathbf{S}_{1}$, and $\mathbf{S}_{2}$ all lie in a plane and precess at the same resonant frequency about the total angular momentum $\mathbf{J}$, which remains fixed in the absence of gravitational radiation. The values of $\theta_{1,2}$ for these resonances can be determined by requiring the first and second time derivatives of $\mathbf{S}_{1} \cdot \mathbf{S}_{2}$ to vanish. This is equivalent to satisfying the algebraic constraint

$$
\begin{aligned}
& \left(\overline{\boldsymbol{\Omega}}_{1} \times \mathbf{S}_{1}\right) \cdot\left[\mathbf{S}_{2} \times\left(\mathbf{L}_{N}+\mathbf{S}_{1}\right)\right] \\
& \quad=\left(\overline{\boldsymbol{\Omega}}_{2} \times \mathbf{S}_{2}\right) \cdot\left[\mathbf{S}_{1} \times\left(\mathbf{L}_{N}+\mathbf{S}_{2}\right)\right] .
\end{aligned}
$$

Since $\mathbf{L}_{N}$ appears in Eq. (3.2) both explicitly and implicitly through $\overline{\boldsymbol{\Omega}}_{1,2}$, the resonant values of $\theta_{1,2}$ vary with the binary separation. This is crucial, as otherwise these oneparameter families of resonances would affect only a small 
portion of the three-dimensional parameter space $\left(\theta_{1}, \theta_{2}, \Delta \phi\right)$ through which generic $\mathrm{BBH}$ configurations evolve. As gravitational radiation slowly extracts angular momentum from the binary on the radiation time $t_{\mathrm{GW}}$, the resonances sweep through a significant portion of the $\left(\theta_{1}, \theta_{2}\right)$ plane. The angular separation $\Delta \phi$ of a generic $\mathrm{BBH}$ is varying on the much shorter precession time $t_{p}$, and thus has a significant chance to closely approach the resonant values $\Delta \phi=0^{\circ}$ or $180^{\circ}$ at some point during the long inspiral. Such generic BBHs will be strongly influenced or even captured by the spin-orbit resonances, as we will see in detail in Sec. IV.

We show the dependence of the spin-orbit resonances on $r$ for maximally spinning BBHs in Figs. 1 and 2. Those resonances with $\Delta \phi=0^{\circ}$ (shown in Fig. 2 of [25]) always have $\theta_{1}<\theta_{2}$, and thus appear below the diagonal $\cos \theta_{1}=$ $\cos \theta_{2}$ in our Figs. 1 and 2. Those resonances with $\Delta \phi=$ $180^{\circ}$ (shown in Fig. 3 of [25]) have $\theta_{1}>\theta_{2}$ and therefore appear above the diagonal in our Figs. 1 and 2. We plot

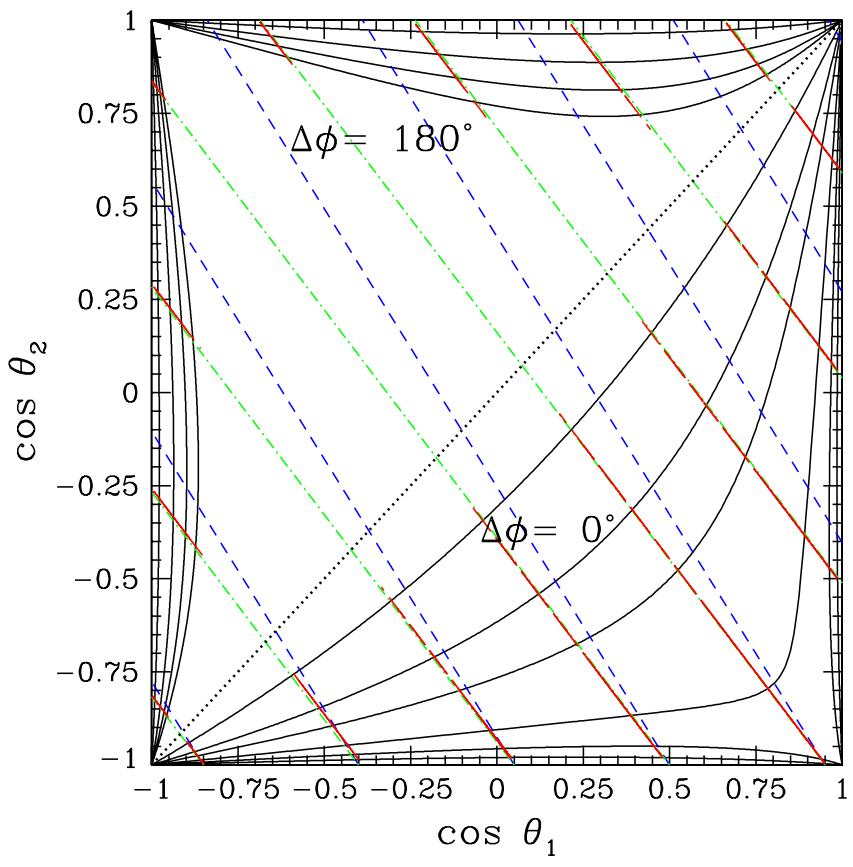

FIG. 1 (color online). Spin-orbit resonances for maximally spinning BBHs with a mass ratio of $q=9 / 11$. The dotted black diagonal indicates where $\theta_{1}=\theta_{2}$. Solid black curves below (above) this diagonal show $\left(\theta_{1}, \theta_{2}\right)$ for the one-parameter families of equilibrium spin configurations with $\Delta \phi=0^{\circ}\left(180^{\circ}\right)$ at different fixed binary separations. Approaching the diagonal from below, these curves correspond to separations $r=$ $1000 M, 500 M, 250 M, 100 M, 50 M, 10 M$. The curves approaching from above correspond to separations $r=250 M, 50 M, 20 M$, $10 M$. The long-dashed red curves show how $\theta_{1,2}$ evolve as members of these resonant families inspiral from $r_{i}=1000 \mathrm{M}$ to $r_{f}=10 M$. The projection $\mathbf{S} \cdot \hat{\mathbf{L}}_{N}$ of the total spin $\mathbf{S}$ onto the orbital angular momentum $\mathbf{L}_{N}$ is constant along the short-dashed blue lines, while the projection $\mathbf{S}_{0} \cdot \hat{\mathbf{L}}_{N}$ of the EOB spin $\mathbf{S}_{0}$ is constant along the dot-dashed green lines.

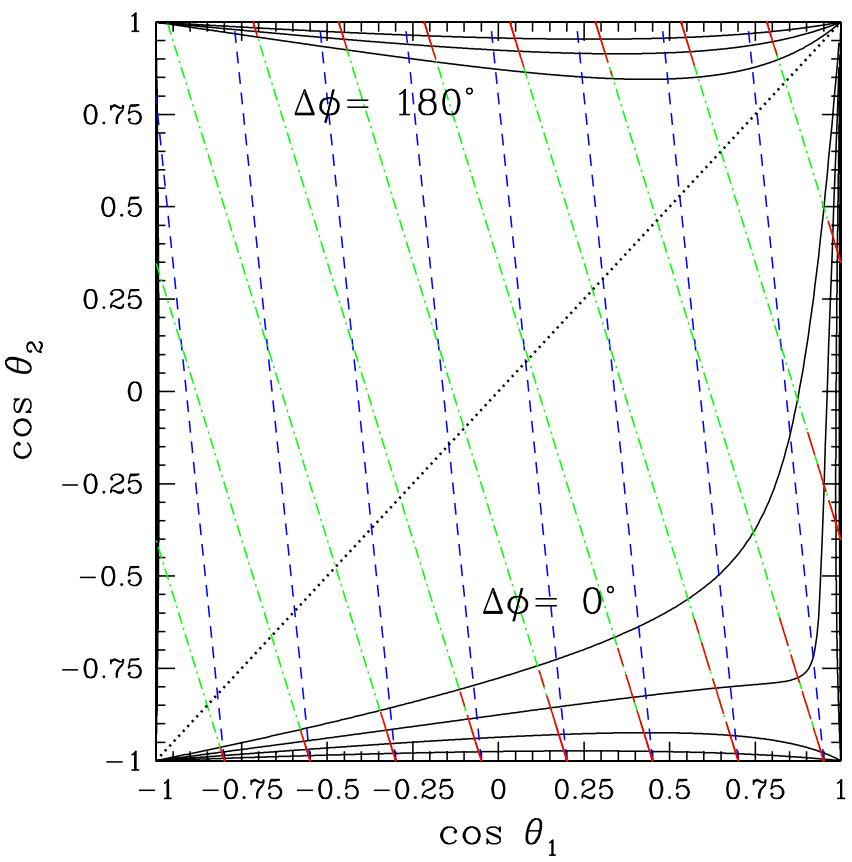

FIG. 2 (color online). Spin-orbit resonances for maximally spinning BBHs with a mass ratio of $q=1 / 3$. Other than the different mass ratio, this figure is very similar to Fig. 1. The solid black curves approaching the diagonal from below correspond to the families of resonant spin configurations at $r=50 M, 20 M$, $10 M, 5 M$, while those approaching from above correspond to separations $r=20 M, 10 M, 5 M$.

$\left(\cos \theta_{1}, \cos \theta_{2}\right)$ rather than $\left(\theta_{1}, \theta_{2}\right)$ like [25] because isotropically oriented spins should have a flat distribution in these variables.

In the limit $r \rightarrow \infty$, so that also $\left|\mathbf{L}_{N}\right| \rightarrow \infty$, the resonant configurations have either $\mathbf{S}_{1}$ or $\mathbf{S}_{2}$ aligned or antialigned with $\mathbf{L}_{N}$ (either $\theta_{1}$ or $\theta_{2}$ equals $0^{\circ}$ or $180^{\circ}$ ). This corresponds to the four edges of the plot in Fig. 1. For smaller fixed values of $\left|\mathbf{L}_{N}\right|$, the values $\left(\theta_{1}, \theta_{2}\right)$ for the oneparameter families of resonant configurations approach the diagonal $\theta_{1}=\theta_{2}$. BBHs in spin-orbit resonances at large values of $\left|\mathbf{L}_{N}\right|$ (large $r$ ) remain resonant as they inspiral. As gravitational radiation carries away angular momentum, $r$ decreases and $\theta_{1,2}$ for individual resonant BBHs evolves toward this diagonal along the red longdashed curves in Fig. 1. For resonances with $\Delta \phi=0^{\circ}$ (those below the diagonal), this evolution aligns the two spins with each other. Symmetry implies that aligning the spins with each other will lead to larger final spins and smaller recoil velocities [38,39].

The projection

$$
\mathbf{S} \cdot \hat{\mathbf{L}}_{N}=S_{1} \cos \theta_{1}+S_{2} \cos \theta_{2}
$$

of the total spin $\mathbf{S} \equiv \mathbf{S}_{1}+\mathbf{S}_{2}$ parallel to the orbital angular momentum is constant along the short-dashed blue lines in Figs. 1 and 2. These blue lines have steeper slopes than the red lines along which the resonant binaries inspiral. This 
implies that the total spin $\mathbf{S}$ becomes antialigned (aligned) with the orbital angular momentum for resonant configurations with $\Delta \phi=0^{\circ}\left(180^{\circ}\right)$, leading to smaller (larger) final spins. The families of resonances with $\Delta \phi=0^{\circ}$ (below the diagonal) sweep through a larger area of the $\left(\cos \theta_{1}, \cos \theta_{2}\right)$ plane as the BBHs inspiral and approach the diagonal more closely. This implies that antialignment may be more effective than alignment, which might explain the "small but statistically significant bias of the distribution towards counter-alignment" in $\mathbf{S} \cdot \hat{\mathbf{L}}_{N}$ noted in Lousto et al. [27]. However, Table IV of [27] indicates that both $\mathbf{S}_{1}$ and $\mathbf{S}_{2}$ individually become antialigned with $\hat{\mathbf{L}}_{N}$, whereas the spin-orbit resonances would align one black hole while antialigning the other. All of the PN evolutions in Lousto et al. [27] begin at separations of $r=50 \mathrm{M}$, which corresponds to the $\Delta \phi=0^{\circ}$ curve in Fig. 1 that is second closest to the diagonal. The resonances sweep through most of the plane below the diagonal at larger separations, suggesting that these short-duration PN evolutions may have failed to capture the full magnitude of the antialignment. We will investigate this possibility in Sec. IV.

Another interesting feature of Figs. 1 and 2 is that the red long-dashed curves along which the BBHs inspiral are nearly parallel to the dot-dashed green lines along which the projection $\mathbf{S}_{0} \cdot \hat{\mathbf{L}}_{N}$ of the effective-one-body (EOB) spin [40]

$$
\mathbf{S}_{0} \equiv(1+q) \mathbf{S}_{1}+\left(1+q^{-1}\right) \mathbf{S}_{2}
$$

is constant. The conservation of this quantity at $2 \mathrm{PN}$ order was first noted in Ref. [33] and follows directly from Eqs. (2.1), (2.2), and (2.5). The conservation of $\mathbf{S}_{0} \cdot \hat{\mathbf{L}}_{N}$ rather than $\mathbf{S} \cdot \hat{\mathbf{L}}_{N}$ itself allows for the possible alignment of the total spin $\mathbf{S}$ discussed in the previous paragraph.

We conclude this section by briefly discussing how the spin-orbit resonances vary with the mass ratio $q$, as can be seen by comparing the $q=9 / 11$ resonances in Fig. 1 with the $q=1 / 3$ resonances in Fig. 2 . The most pronounced differences are that the $q=1 / 3$ resonances sweep away from the edges of the $\left(\cos \theta_{1}, \cos \theta_{2}\right)$ plane at much smaller values of the separation $r$ and do not approach the diagonal as closely. This is consistent with the decreasing value of $r_{\text {lock }}$ in Eq. (1.4) as $q \rightarrow 0$. In this limit both $t_{p}$ and $t_{\mathrm{GW}}$ are proportional to $q^{-1}$, implying that generic BBHs will be less likely to be affected by the resonances as they sweep through the plane over a smaller range in $r$. BBHs already in a resonant configuration will also be less affected since the resonant curves do not approach the diagonal as closely. The red long-dashed curves showing the inspiral of resonant configurations have steeper slopes for $q=1 / 3$, consistent with the larger black hole being immune to its smaller companion in the limit $q \rightarrow 0$. This seems to contradict the puzzling result presented in Table IV of Lousto et al. [27] that it is the smaller companion that remains randomly distributed during the inspiral. We will examine this behavior as well in the next section.

\section{SPIN ALIGNMENT}

In this section, we examine the extent to which the spins of generic (i.e. misaligned) BBH configurations become aligned with the orbital angular momentum and each other as the BBHs inspiral from $r_{i}=1000 M$ to $r_{f}=10 M$. Although we use maximally spinning BBHs to demonstrate this alignment, the magnitude of the alignment is comparable for all BBHs with $\chi_{1,2} \gtrsim 0.5$ as shown in Fig. 11 of [25]. We first consider initial spin configurations given by a uniform $10 \times 10 \times 10$ grid evenly spaced in $\left(\cos \theta_{1}, \cos \theta_{2}, \Delta \phi\right)$. This distribution is isotropic and would be expected in the absence of an astrophysical mechanism to align the spins. BBHs with isotropically oriented spins might form in gas-poor mergers of SBHs and mergers of stellar-mass black holes in dense clusters.

In Fig. 3, we show how the distribution of $\left(\cos \theta_{1}, \cos \theta_{2}\right)$ evolves as maximally spinning $\mathrm{BBHs}$ with a mass ratio $q=9 / 11$ inspiral from slightly beyond $r_{i}=1000 M$ to $r_{f}=10 M$. The top left panel shows our initial evenly

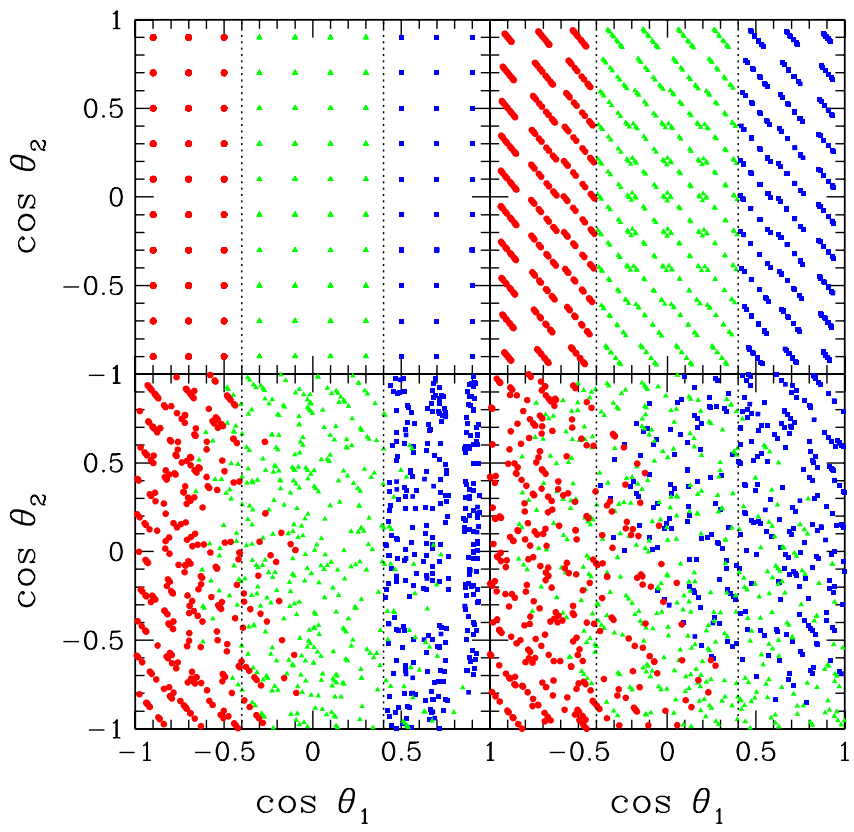

FIG. 3 (color online). Distributions of $\left(\cos \theta_{1}, \cos \theta_{2}\right)$ at different separations $r$ for 1000 initially isotropic maximally spinning BBHs with a mass ratio $q=9 / 11$. The top left panel shows the initial $10 \times 10 \times 10$ grid, evenly spaced in $\left(\cos \theta_{1}, \cos \theta_{2}, \Delta \phi\right)$. The dotted vertical lines show $\cos \theta_{1}= \pm 0.4$. The 300 blue squares initially have $\cos \theta_{1}>0.4$, the 400 green triangles initially have $-0.4<\cos \theta_{1}<0.4$, and the 300 red circles initially have $\cos \theta_{1}<-0.4$. The values of $\left(\theta_{1}, \theta_{2}\right)$ for these BBHs are shown in the top right, bottom left, and bottom right panels after they have inspiraled to separations of $r=1000 M, 100 M$, and $10 M$, respectively. 
spaced $10 \times 10 \times 10$ grid. The points are colored to indicate their initial value of $\cos \theta_{1}$ : blue squares begin with $\cos \theta_{1}>0.4\left(\theta_{1} \lesssim 66^{\circ}\right)$, green triangles with $-0.4<$ $\cos \theta_{1}<0.4$, and red circles with $\cos \theta_{1}<-0.4$. The dotted vertical lines $\cos \theta= \pm 0.4$ denote these boundaries. Only 100 points are visible in the top left panel, as the different values of $\Delta \phi$ cannot be distinguished in this twodimensional projection. Spin precession reveals all 1000 points after the BBHs have inspiraled to $r_{i}=1000 \mathrm{M}$ as seen in the top right panel. Notice that the spins of all 1000 BBHs precess in a way that conserves the projection of $\mathbf{S}_{0}$ onto $\hat{\mathbf{L}}_{N}$ (parallel to the dot-dashed green lines in Fig. 1). This is not a special feature of the spin-orbit resonances, but occurs for generically oriented spins as well. These generic spin configurations do not individually preserve $\left(\cos \theta_{1}, \cos \theta_{2}\right)$ over a precession time $t_{p}$ like the resonant configurations do, but they do preserve the combination $\mathbf{S}_{0} \cdot \hat{\mathbf{L}}_{N}$. This precession continues as the BBHs inspiral to $r=100 M$ and $r_{f}=10 M$ as shown in the bottom left and bottom right panels of Fig. 3. By the time they reach $r_{f}=$ $10 M$ the green points have diffused to fill most of the $\left(\cos \theta_{1}, \cos \theta_{2}\right)$ plane, while the blue (red) points have diffused into the upper right (lower left) portion of the middle $-0.4<\cos \theta_{1}<0.4$ region. The bottom right panel, if the points had not been colored, would reproduce Fig. 1 of Bogdanovic et al. [23] and therefore support their conclusion that isotropically distributed spins remain isotropic as they inspiral. However, the colors reveal that PN evolution can drastically alter spin distributions that have been partially aligned by a circumbinary disk. For example, if the spin of the more massive black hole was aligned so that $\cos \theta_{1}>0.4$ at $r_{i}=1000 M$ (shown by our blue points), by the time the binary reached $r_{f}=$ $10 M$ the larger spin could easily lie in the orbital plane and thus give rise to a smaller final spin and potentially large "superkick" $[15,16]$.

For comparison, we show the inspiral of the same $10 \times$ $10 \times 10$ grid of maximally spinning BBHs with a mass ratio $q=1 / 3$ in Fig. 4. The points diffuse along the steeper lines that preserve $\mathbf{S}_{0} \cdot \hat{\mathbf{L}}_{N}$ for this less equal mass ratio. This inhibits their ability to diffuse across the $\cos \theta_{1}=$ \pm 0.4 boundaries, again shown by the vertical dotted lines. Even at $r_{f}=10 M$ only a few points have trickled between the three regions. Since the spin of the more massive black hole remains aligned with the orbital angular momentum, one would expect a large final spin and an absence of superkicks for such small mass ratios. We examine in detail how spin alignment affects recoil-velocity distributions in Ref. [41].

In Fig. 5 we show how the joint probability distribution function for $\Delta \phi$ and $\cos \theta_{12}$ evolves for our evenly spaced $10 \times 10 \times 10$ grid of initially isotropic $\mathrm{BBH}$ spin configurations. As defined in the Introduction, $\cos \theta_{12}$ is the cosine of the angle between $\mathbf{S}_{1}$ and $\mathbf{S}_{2}$. It can be expressed in terms of the individual spin angles as

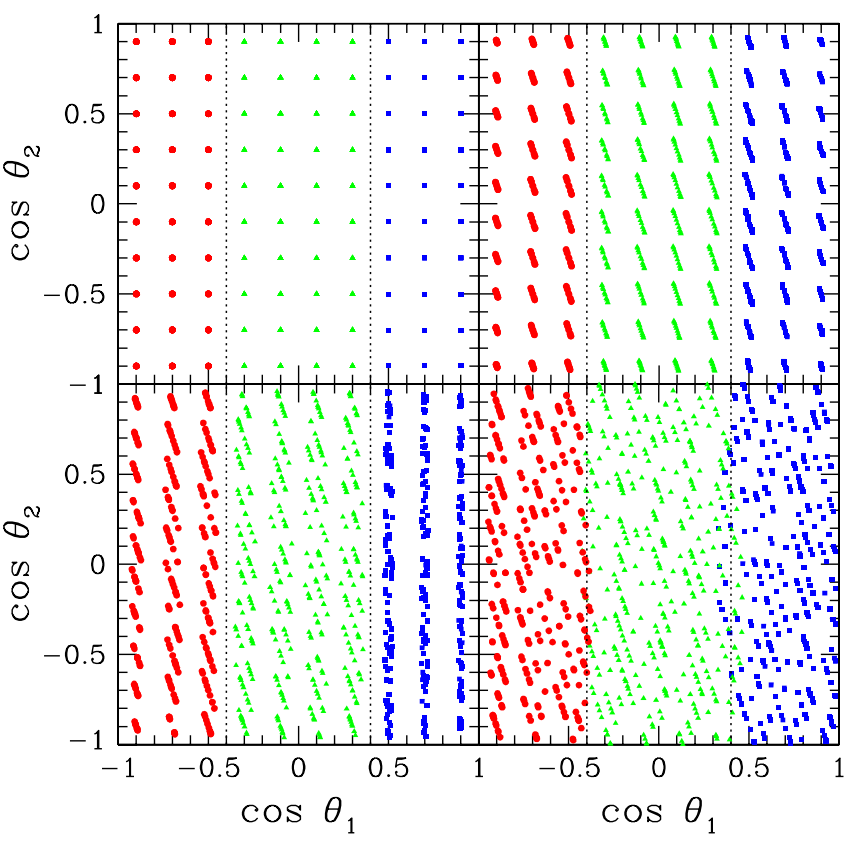

FIG. 4 (color online). Distributions of $\left(\cos \theta_{1}, \cos \theta_{2}\right)$ at different separations $r$ for 1000 initially isotropic maximally spinning BBHs with a mass ratio $q=1 / 3$. The different panels, points, and lines are the same as those given for $q=9 / 11$ in Fig. 3 .

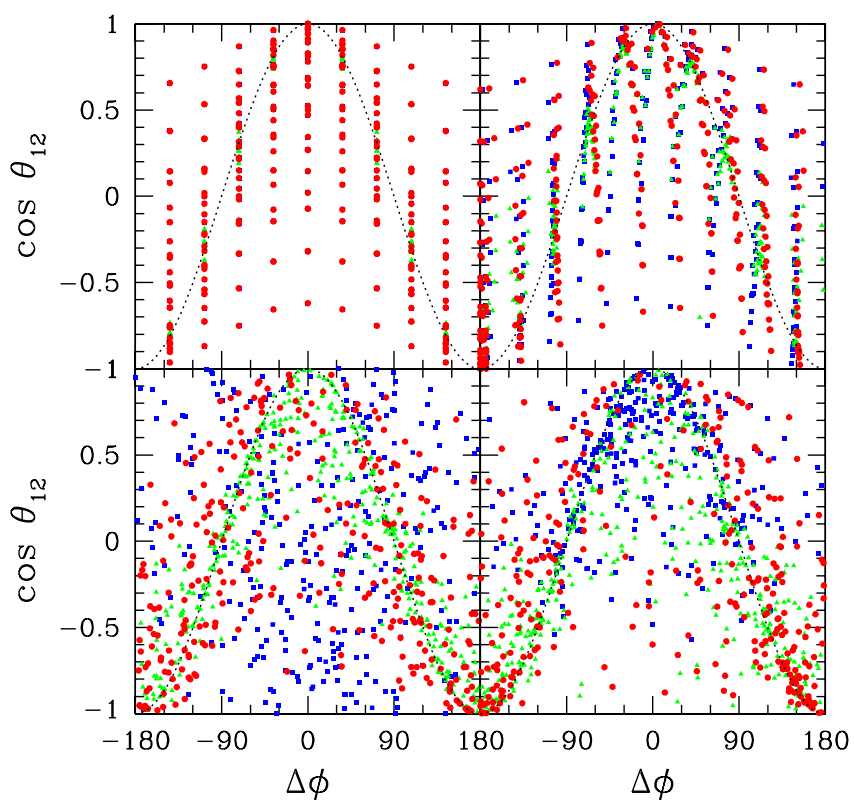

FIG. 5 (color online). Distributions of $\left(\Delta \phi, \cos \theta_{12}\right)$ at different separations $r$ for 1000 initially isotropic maximally spinning BBHs with a mass ratio $q=9 / 11$. The top left panel shows the initial $10 \times 10 \times 10$ grid of $\mathrm{BBH}$ spin configurations, evenly spaced in $\left(\cos \theta_{1}, \cos \theta_{2}, \Delta \phi\right)$. This distribution is peaked about the curve $\cos \theta_{12}=\cos \Delta \phi$ shown by the dotted curve. The points are colored according to their initial values of $\cos \theta_{1}$ as in Fig. 3. The top right, bottom left, and bottom right panels show the distribution evolves after the BBHs have inspiraled to $r=1000 M, 100 M$, and 10M, respectively, also as in Fig. 3 . 


$$
\cos \theta_{12}=\sin \theta_{1} \sin \theta_{2} \cos \Delta \phi+\cos \theta_{1} \cos \theta_{2},
$$

and has a flat distribution between -1 and 1 for isotropic, uncorrelated spins such as those given by our $10 \times 10 \times$ 10 grid. However, as seen in Eq. (4.1), the values of $\cos \theta_{12}$ and $\cos \Delta \phi$ are correlated; for a given value of $\Delta \phi$ the distribution of $\cos \theta_{12}$ is peaked about $\cos \Delta \phi$ for flat distributions of $\cos \theta_{1}$ and $\cos \theta_{2}$. This can be seen in Fig. 5 from the clustering of points about the curve $\cos \theta_{12}=$ $\cos \Delta \phi$. Although $\cos \theta_{12}$ and $\cos \Delta \phi$ are correlated even for isotropic spins, geometry implies that both are initially uncorrelated with the value of $\cos \theta_{1}$. This is revealed by the identical distributions of the red circles, green triangles, and blue squares in the top left panel of Fig. 5 to within the resolution of our grid. These distributions do not remain identical as the BBHs inspiral from $r_{i}=1000 M$ to $r_{f}=$ $10 M$. Influenced by the $\Delta \phi=0^{\circ}$ spin-orbit resonances below the diagonal in Fig. 1, the blue points become concentrated about $\Delta \phi=0^{\circ}, \cos \theta_{12}=1$ by the time they reach $r_{f}$. The red points, similarly influenced by the $\Delta \phi= \pm 180^{\circ}$ resonances above the diagonal in Fig. 1, become concentrated about $\Delta \phi= \pm 180^{\circ}, \cos \theta_{12}=-1$. The effect of this spin alignment on the spin of the final black hole will be explored in detail in the next section, while the effect on recoil velocities is examined in Ref. [41]. Qualitatively, alignment of the spins with each other $\left(\cos \theta_{12} \rightarrow 1\right)$ increases the final spin and reduces the recoil velocity, while antialignment $\left(\cos \theta_{12} \rightarrow-1\right)$ does the opposite.

The magnitude of this spin alignment is greatly reduced for smaller mass ratios as seen in Fig. 6 for the case $q=$

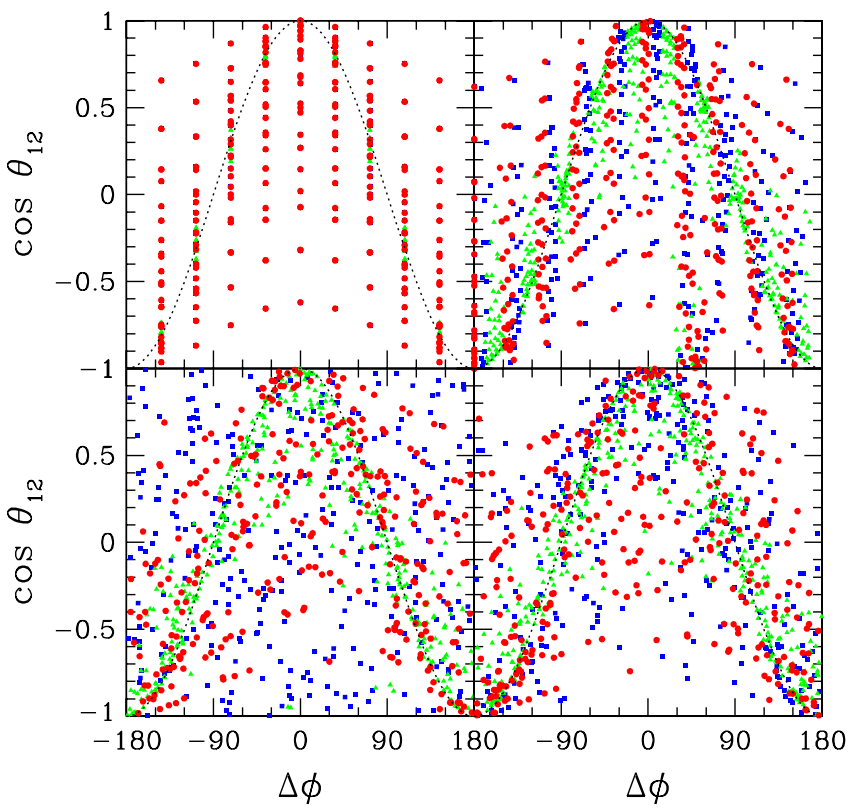

FIG. 6 (color online). Distributions of $\left(\Delta \phi, \cos \theta_{12}\right)$ at different separations $r$ for 1000 initially isotropic maximally spinning BBHs with a mass ratio $q=1 / 3$. The different panels, points, and lines are the same as those given for $q=9 / 11$ in Fig. 5 .

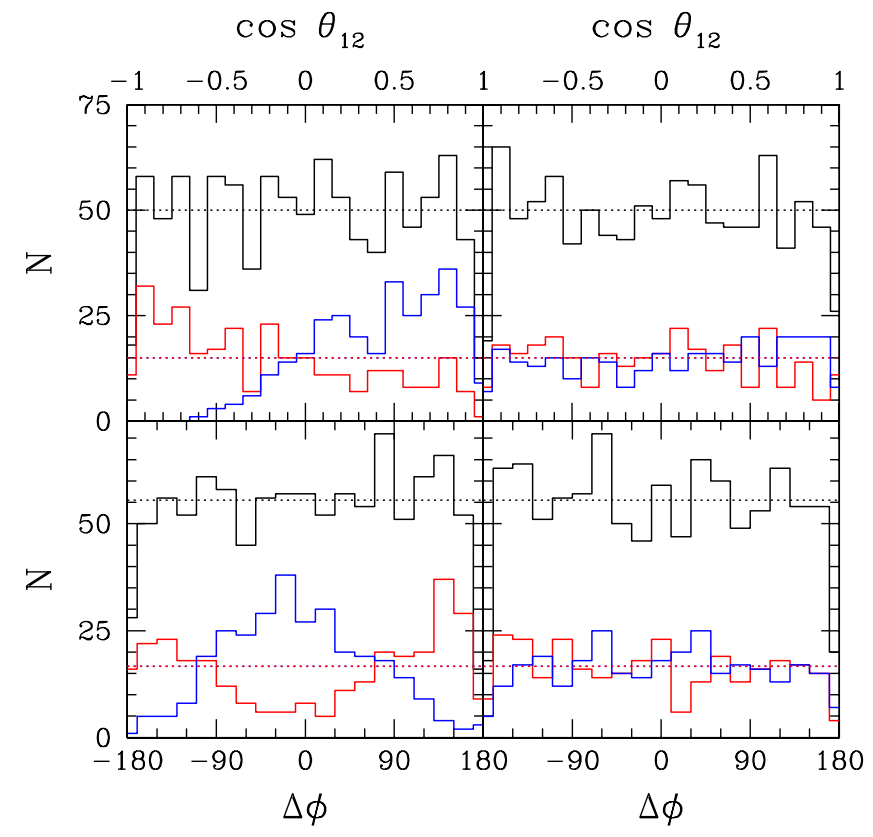

FIG. 7 (color online). Histograms of $\cos \theta_{12}$ and $\Delta \phi$ for BBHs with initially isotropic spins. The two left panels are for the mass ratio $q=9 / 11$, while the two right panels are for $q=1 / 3$. The two top panels give the distribution of $\cos \theta_{12}$, while the two bottom panels give the distribution of $\Delta \phi$. The black curves are for all $1000 \mathrm{BBHs}$ in the $10 \times 10 \times 10$ grid discussed in the text, while the blue (red) curves correspond to the blue (red) points in Figs. 3-6 with initial values $\cos \theta_{1}>0.4\left(\cos \theta_{1}<-0.4\right)$. The horizontal dotted lines show the initially flat distributions, while the solid lines show the distributions at $r=10 \mathrm{M}$.

$1 / 3$. Although the clustering of all the points about $\cos \theta_{12}=\cos \Delta \phi$ is again apparent, the distributions of the red, green, and blue points remain similar all the way down to $r_{f}=10 M$ as seen in the lower right panel. The weaker influence of the spin-orbit resonances for $q=1 / 3$ follows from the smaller value of $r_{\text {lock }}$ in Eq. (1.4) and is similarly reflected by the smaller fraction of the $\left(\cos \theta_{1}, \cos \theta_{2}\right)$ plane occupied by the resonant curves in Fig. 2.

We have provided histograms of $\cos \theta_{12}$ and $\Delta \phi$ in Fig. 7 to clarify the differences between Figs. 5 and 6 . We see that the distributions of $\cos \theta_{12}$ and $\Delta \phi$ are initially flat for both mass ratios, but evolve considerably for $q=9 / 11$ while remaining nearly flat for $q=1 / 3$ within the limits set by Poisson fluctuations. The open blue (red) curves in the left panels of Fig. 7 clearly show distributions peaked at $\cos \theta_{12}=1, \Delta \phi=0^{\circ} \quad\left(\cos \theta_{12}=-1, \quad \Delta \phi= \pm 180^{\circ}\right)$. Such trends are barely noticeable in the right panels. We will explore the implications of these findings for the final spins in the next section.

\section{FINAL SPIN DISTRIBUTIONS}

Several attempts have been made to predict the final dimensionless spin $\boldsymbol{\chi}_{f}$ of the black hole resulting from a 
BBH merger. Initial attempts focused on finding simple phenomenological fitting formulas for the final spin resulting from nonspinning, unequal-mass $\mathrm{BBH}$ merger simulations [42-44]. A group at the Albert Einstein Institute (AEI) developed a fitting formula that provides the magnitude and direction of $\boldsymbol{\chi}_{f}$ in terms of the initial spins $\boldsymbol{\chi}_{1}, \boldsymbol{\chi}_{2}$ and the mass ratio $q$ [45-47]. They assumed that the final spin magnitude could be expressed as a polynomial in $\chi_{1}$, $\chi_{2}$, and the symmetric mass ratio $\eta$, and then made some additional assumptions about the symmetries of this polynomial dependence and how energy and angular momentum are radiated to reduce the number of terms in their expression. The coefficients of the remaining terms were calibrated using numerical-relativity (NR) simulations of $\mathrm{BBH}$ mergers in which the initial spins were either aligned or antialigned with the orbital angular momentum. We shall refer to this older AEI formula as "AEIo." A more recent paper [30] by members of this group uses newer NR simulations to recalibrate their coefficients, and replaces earlier assumptions with the conjecture that the final spin points in the direction of the total angular momentum of the initial BBH at any separation. For consistency, this requires the further assumption that angular momentum is always radiated in the direction of the total angular momentum. We shall refer to this newer AEI formula as "AEIn." An alternative fitting formula was proposed by a group at Florida Atlantic University (FAU) [48]. Following the procedure outlined in [38,39], the FAU group performed 10 equal-mass misaligned simulations to calibrate the coefficients of fitting formulas for the Cartesian components of $\boldsymbol{\chi}_{f}$. They then made additional assumptions about the mass-ratio dependence of these formulas and found good agreement between their predictions and independent NR simulations with mass ratios as small as $q=$ $5 / 8$. We shall refer to the formula of this group as "FAU." The Rochester Institute of Technology (RIT) group proposed yet another fitting formula during the preparation of this paper [49]. This formula includes higher-order terms in the initial spins that may ultimately be needed to describe future high-accuracy NR simulations. However, current simulations are inadequate to calibrate all the terms appearing in the RIT formula, so we will not consider its predictions in this paper.

Other groups have predicted final spins by extrapolating analytical test-particle calculations to finite mass ratios, rather than calibrating fitting formulas with NR simulations. Buonanno, Kidder, and Lehner (BKL) [50] derived a formula for the final spin by assuming, as is true in the testparticle limit, that the angular momentum radiated during the inspiral stage of a BBH merger exceeds that radiated during the plunge and ringdown. Using this assumption, they equated the final spin with the total angular momentum $\mathbf{J}=\mathbf{L}_{\text {ISCO }}+\mathbf{S}_{1}+\mathbf{S}_{2}$, where $\mathbf{L}_{\text {ISCO }}$ is the orbital angular momentum at the innermost stable circular orbit (ISCO) of a test particle of mass $\eta M$ orbiting a black hole of mass $M$ and dimensionless spin $\boldsymbol{\chi}_{f}$ equal to that of the final black hole. This counterintuitive but inspired choice correctly provides $\boldsymbol{\chi}_{f} \rightarrow \boldsymbol{\chi}_{1}$ in the $q \rightarrow 0$ limit and respects the symmetry of $\mathrm{BBH}$ mergers under exchange of the labels of the two black holes. Though derived only from test-particle calculations, the BKL formula is remarkably successful at predicting final spins even for equalmass BBH mergers. Kesden [51] slightly modified the BKL spin formula to account for the energy radiated during the inspiral stage of the merger. This change makes the formula accurate to linear order in $q$ in the test-particle limit. It generically increases the magnitude of the predicted dimensionless final spin by reducing the predicted final mass $m_{f}$ below $M$ in the denominator of the expression $\boldsymbol{\chi}_{f}=S_{f} / m_{f}^{2}$. This increase improves the agreement

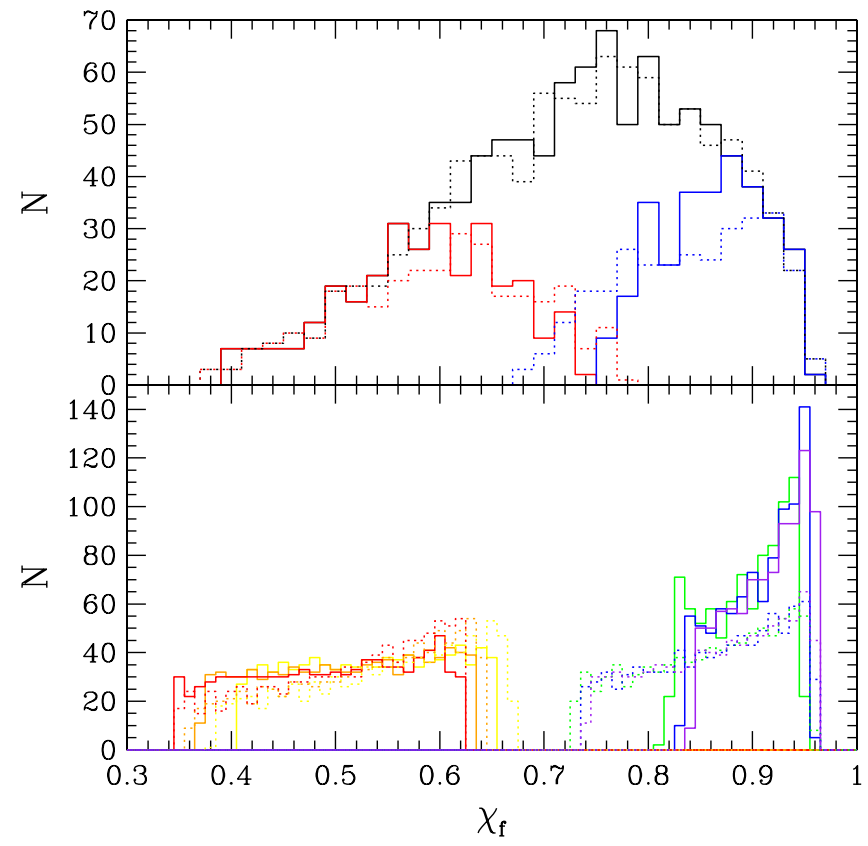

FIG. 8 (color online). Top panel: Histogram of the final spin $\chi_{f}$ predicted by the AEIn formula for $1000 \mathrm{BBHs}$ with mass ratio $q=9 / 11$ and isotropically distributed spins at $r_{i}=$ $1000 \mathrm{M}$. The blue curves show the subset of $300 \mathrm{BBHs}$ with the lowest initial values of $\theta_{1}$, while the red curves show the subset of $300 \mathrm{BBHs}$ with the highest initial values of $\theta_{1}$. The solid curves show the predicted spins if the AEIn formula is applied at $r_{f}=10 M$ after the BBHs have inspiraled to this separation according to the equations of Sec. II. The dotted curves show the predicted spins if the AEIn formula is applied to the initial distribution at $r_{i}=1000 M$. Bottom panel: Histograms of the predicted final spins for 6 sets of BBH mergers with $q=9 / 11$, and flat distributions in $\cos \theta_{2}$ and $\Delta \phi$ at $r_{i}=$ $1000 \mathrm{M}$. The red, orange, yellow, green, blue, and purple curves have $\theta_{1}=170^{\circ}, 160^{\circ}, 150^{\circ}, 30^{\circ}, 20^{\circ}$, and $10^{\circ}$, respectively. As in the top panel the final spins predicted by applying the AEIn formula at $r_{i}=1000 M$ are shown by dotted curves, while allowing the $\mathrm{BBHs}$ to inspiral to $r_{f}=10 \mathrm{M}$ before applying the formula leads to the spins shown by the solid curves. 
with NR simulations of nonspinning BBH mergers, but leads to somewhat larger final spins than the other formulas for mergers of maximally spinning BBHs, such as those considered in this paper. The predictions of this formula are referred to as "Kes" in this paper.

We now present the predictions of the spin formulas summarized above for various distributions of BBH spins that are allowed to inspiral from $r_{i}=1000 M$ to $r_{f}=10 M$.

\section{A. Spin magnitudes}

In the top panel of Fig. 8, we show the final spin magnitude $\chi_{f}$ predicted by the AEIn formula for the evenly spaced $10 \times 10 \times 10$ grid of maximally spinning $\mathrm{BBHs}$ with $q=9 / 11$ described in Sec. IV. The other spin formulas give very similar results; the mean and variance of the final spin distributions predicted by the other formulas for some of the initial distributions described below are provided in Table I. As in Figs. 3-7, the black curves in Fig. 8 refer to all $1000 \mathrm{BBHs}$, the blue curves to the subset of $300 \mathrm{BBHs}$ with the lowest values of $\theta_{1}$, and the red curves to the subset of $300 \mathrm{BBHs}$ with the highest values of $\theta_{1}$. The dotted curves give the final spin distribution predicted for the BBH spin configurations at their initial separation $r_{i}=1000 M$, while the solid curves give the final spin distribution predicted when these same BBHs are allowed to inspiral to $r_{f}=10 M$ according to the PN

TABLE I. Mean and standard deviation of the final spin magnitudes predicted for different sets of maximally spinning BBH mergers. The first column lists the formulas used to predict the final spins, as described in Sec. V. The second column gives the mass ratio $q$. Each set of BBHs begins at $r_{i}=1000 M$ with the indicated value of $\theta_{1}$ and flat distributions of $\cos \theta_{2}$ and $\Delta \phi$. The third, fourth, and fifth columns show the mean and deviation expected if the $\mathrm{BBH}$ spins do not precess, thus maintaining their initial distributions at $r_{i}=1000 M$ until merger. The sixth, seventh, and eighth columns assume that the spins precess according to the PN equations of Sec. II as they inspiral to $r_{f}=10 M$, at which separation we apply the spin formulas.

\begin{tabular}{|c|c|c|c|c|c|c|c|}
\hline Model & $q$ & $\theta_{1}=10^{\circ}$ & $\begin{array}{c}r_{i}=1000 M \\
\theta_{1}=20^{\circ}\end{array}$ & $\theta_{1}=30^{\circ}$ & $\theta_{1}=10^{\circ}$ & $\begin{array}{c}r_{f}=10 M \\
\theta_{1}=20^{\circ}\end{array}$ & $\theta_{1}=30^{\circ}$ \\
\hline AEIn & $9 / 11$ & $0.867 \pm 0.064$ & $0.863 \pm 0.065$ & $0.857 \pm 0.066$ & $0.914 \pm 0.034$ & $0.905 \pm 0.036$ & $0.892 \pm 0.038$ \\
\hline AEIo & $9 / 11$ & $0.866 \pm 0.063$ & $0.863 \pm 0.064$ & $0.856 \pm 0.065$ & $0.912 \pm 0.034$ & $0.904 \pm 0.036$ & $0.891 \pm 0.038$ \\
\hline FAU & $9 / 11$ & $0.873 \pm 0.059$ & $0.868 \pm 0.060$ & $0.861 \pm 0.061$ & $0.909 \pm 0.035$ & $0.901 \pm 0.037$ & $0.888 \pm 0.039$ \\
\hline BKL & $9 / 11$ & $0.862 \pm 0.067$ & $0.858 \pm 0.068$ & $0.851 \pm 0.070$ & $0.905 \pm 0.037$ & $0.898 \pm 0.039$ & $0.884 \pm 0.042$ \\
\hline Kes & $9 / 11$ & $0.901 \pm 0.072$ & $0.896 \pm 0.073$ & $0.889 \pm 0.075$ & $0.950 \pm 0.038$ & $0.941 \pm 0.041$ & $0.927 \pm 0.044$ \\
\hline AEIn & $2 / 3$ & $0.886 \pm 0.052$ & $0.882 \pm 0.053$ & $0.875 \pm 0.054$ & $0.922 \pm 0.030$ & $0.914 \pm 0.031$ & $0.900 \pm 0.034$ \\
\hline AEIo & $2 / 3$ & $0.886 \pm 0.052$ & $0.882 \pm 0.052$ & $0.876 \pm 0.054$ & $0.922 \pm 0.030$ & $0.914 \pm 0.031$ & $0.900 \pm 0.034$ \\
\hline FAU & $2 / 3$ & $0.901 \pm 0.043$ & $0.895 \pm 0.044$ & $0.886 \pm 0.046$ & $0.924 \pm 0.029$ & $0.915 \pm 0.030$ & $0.901 \pm 0.031$ \\
\hline BKL & $2 / 3$ & $0.882 \pm 0.052$ & $0.878 \pm 0.053$ & $0.870 \pm 0.054$ & $0.914 \pm 0.031$ & $0.906 \pm 0.032$ & $0.893 \pm 0.035$ \\
\hline Kes & $2 / 3$ & $0.921 \pm 0.056$ & $0.917 \pm 0.057$ & $0.909 \pm 0.059$ & $0.958 \pm 0.031$ & $0.949 \pm 0.034$ & $0.935 \pm 0.037$ \\
\hline AEIn & $1 / 3$ & $0.950 \pm 0.025$ & $0.946 \pm 0.025$ & $0.938 \pm 0.026$ & $0.957 \pm 0.023$ & $0.951 \pm 0.023$ & $0.941 \pm 0.022$ \\
\hline AEIo & $1 / 3$ & $0.958 \pm 0.025$ & $0.953 \pm 0.026$ & $0.944 \pm 0.026$ & $0.964 \pm 0.023$ & $0.958 \pm 0.023$ & $0.947 \pm 0.022$ \\
\hline FAU & $1 / 3$ & $0.972 \pm 0.013$ & $0.964 \pm 0.014$ & $0.951 \pm 0.016$ & $0.975 \pm 0.012$ & $0.966 \pm 0.012$ & $0.953 \pm 0.011$ \\
\hline BKL & $1 / 3$ & $0.931 \pm 0.020$ & $0.927 \pm 0.020$ & $0.921 \pm 0.021$ & $0.936 \pm 0.018$ & $0.931 \pm 0.018$ & $0.923 \pm 0.018$ \\
\hline Kes & $1 / 3$ & $0.968 \pm 0.021$ & $0.965 \pm 0.022$ & $0.958 \pm 0.023$ & $0.974 \pm 0.019$ & $0.970 \pm 0.019$ & $0.962 \pm 0.020$ \\
\hline Model & $q$ & $\theta_{1}=150^{\circ}$ & $\theta_{1}=160^{\circ}$ & $\theta_{1}=170^{\circ}$ & $\theta_{1}=150^{\circ}$ & $\theta_{1}=160^{\circ}$ & $\theta_{1}=170^{\circ}$ \\
\hline AEIn & $9 / 11$ & $0.551 \pm 0.080$ & $0.527 \pm 0.080$ & $0.511 \pm 0.079$ & $0.535 \pm 0.072$ & $0.510 \pm 0.076$ & $0.493 \pm 0.080$ \\
\hline AEIo & $9 / 11$ & $0.551 \pm 0.080$ & $0.527 \pm 0.080$ & $0.512 \pm 0.079$ & $0.535 \pm 0.072$ & $0.510 \pm 0.077$ & $0.493 \pm 0.080$ \\
\hline FAU & $9 / 11$ & $0.542 \pm 0.076$ & $0.520 \pm 0.076$ & $0.506 \pm 0.076$ & $0.530 \pm 0.070$ & $0.507 \pm 0.074$ & $0.492 \pm 0.076$ \\
\hline BKL & $9 / 11$ & $0.514 \pm 0.088$ & $0.488 \pm 0.087$ & $0.471 \pm 0.086$ & $0.496 \pm 0.078$ & $0.468 \pm 0.083$ & $0.449 \pm 0.087$ \\
\hline Kes & $9 / 11$ & $0.531 \pm 0.091$ & $0.504 \pm 0.090$ & $0.486 \pm 0.089$ & $0.512 \pm 0.081$ & $0.483 \pm 0.087$ & $0.463 \pm 0.091$ \\
\hline AEIn & $2 / 3$ & $0.500 \pm 0.067$ & $0.467 \pm 0.066$ & $0.445 \pm 0.065$ & $0.490 \pm 0.057$ & $0.456 \pm 0.062$ & $0.432 \pm 0.065$ \\
\hline AEIo & $2 / 3$ & $0.499 \pm 0.067$ & $0.466 \pm 0.067$ & $0.444 \pm 0.066$ & $0.489 \pm 0.057$ & $0.455 \pm 0.062$ & $0.432 \pm 0.065$ \\
\hline FAU & $2 / 3$ & $0.490 \pm 0.060$ & $0.460 \pm 0.060$ & $0.441 \pm 0.059$ & $0.483 \pm 0.053$ & $0.452 \pm 0.056$ & $0.432 \pm 0.059$ \\
\hline BKL & $2 / 3$ & $0.465 \pm 0.072$ & $0.430 \pm 0.071$ & $0.405 \pm 0.070$ & $0.454 \pm 0.060$ & $0.416 \pm 0.066$ & $0.390 \pm 0.070$ \\
\hline Kes & $2 / 3$ & $0.480 \pm 0.075$ & $0.442 \pm 0.071$ & $0.417 \pm 0.072$ & $0.468 \pm 0.063$ & $0.428 \pm 0.068$ & $0.401 \pm 0.072$ \\
\hline AEIn & $2 / 3$ & $0.324 \pm 0.034$ & $0.233 \pm 0.034$ & $0.151 \pm 0.032$ & $0.323 \pm 0.013$ & $0.231 \pm 0.015$ & $0.145 \pm 0.021$ \\
\hline AEIo & $2 / 3$ & $0.321 \pm 0.034$ & $0.230 \pm 0.034$ & $0.146 \pm 0.032$ & $0.319 \pm 0.012$ & $0.227 \pm 0.015$ & $0.140 \pm 0.021$ \\
\hline FAU & $2 / 3$ & $0.301 \pm 0.026$ & $0.222 \pm 0.026$ & $0.154 \pm 0.024$ & $0.300 \pm 0.016$ & $0.220 \pm 0.018$ & $0.151 \pm 0.020$ \\
\hline BKL & $2 / 3$ & $0.315 \pm 0.034$ & $0.222 \pm 0.034$ & $0.136 \pm 0.032$ & $0.313 \pm 0.011$ & $0.219 \pm 0.013$ & $0.130 \pm 0.019$ \\
\hline Kes & $2 / 3$ & $0.322 \pm 0.035$ & $0.227 \pm 0.035$ & $0.139 \pm 0.033$ & $0.320 \pm 0.012$ & $0.224 \pm 0.014$ & $0.133 \pm 0.019$ \\
\hline
\end{tabular}


evolution described in Sec. II. The AEIn formula is unique in that it claims to accurately predict final spins at all separations; separations as large as $r=2 \times 10^{4} M$ were considered in [30]. The other fitting formulas were intended to apply at $r_{\mathrm{NR}} \simeq 10 \mathrm{M}$, the starting point for the NR simulations with which their coefficients were calibrated. The BKL and Kes formulas were designed for use at the ISCO. Although strictly speaking the formulas other than AEIn cannot be applied to widely separated BBHs, one can imagine that the BBHs inspiral to $r_{f}=10 M$ without spin precession where these formulas are valid. It is in this sense that we consider the predictions of these other formulas when we claim in this section to apply them to the $\mathrm{BBH}$ spin configuration at $r_{i}=1000 M$.

The dotted and solid black curves in the top panel of Fig. 8 are identical to each other within the Poisson noise of our limited number of $\mathrm{BBH}$ inspirals, confirming the finding of Refs. [23,26,27] that isotropic distributions of $\mathrm{BBH}$ spins remain nearly isotropic as they inspiral. Even at $r_{i}=1000 M$, the blue (red) subset of spin configurations yields the largest (smallest) predicted final spins, because for these configurations the spin of the more massive black hole is aligned (antialigned) with the orbital angular momentum. The spin-orbit resonances further enhance (reduce) the final spins predicted for these subsets by aligning (antialigning) the $\mathrm{BBH}$ spins with each other during the inspiral for small (large) initial values of $\theta_{1}$. As a result, the solid blue (red) distribution at $r_{f}=10 M$ has a larger (smaller) mean final spin than the initial dotted distribution at $r_{i}=1000 M$. This can be seen in the displacement of predicted final spins for the colored subsets away from $\chi_{f} \simeq 0.75$ toward larger and smaller values.

To clarify the magnitude of this effect, we have performed 6 additional sets of BBH inspirals, each of which consists of a fixed value of $\theta_{1}$ and a $30 \times 30$ grid evenly spaced in $\cos \theta_{2}$ and $\Delta \phi$. Three of these sets have the spin of the more massive black hole nearly aligned with the orbital angular momentum $\left(\theta_{1}=10^{\circ}, 20^{\circ}, 30^{\circ}\right)$, while the other 3 sets have $\boldsymbol{\chi}_{1}$ nearly antialigned with $\mathbf{L}_{N}\left(\theta_{1}=\right.$ $\left.150^{\circ}, 160^{\circ}, 170^{\circ}\right)$. The choice of aligned distributions was partly motivated by the finding of Ref. [17] that accretion torques will align $\mathrm{BBH}$ spins to within $10^{\circ}\left(30^{\circ}\right)$ of the orbital angular momentum for a cold (hot) disk. The predicted final spins for these distributions, at both $r_{i}=$ $1000 M$ and $r_{f}=10 M$, are shown in the bottom panel of Fig. 8. The final spins for the initially aligned $\left(\theta_{1} \leq 30^{\circ}\right)$ $\mathrm{BBH}$ distributions are significantly larger when predicted at $r_{f}=10 M$ than at $r_{i}=1000 M$, undermining the claim of [30] that the AEIn formula can accurately predict final spins at large separations without the need for PN evolutions. The predicted final spins for the initially antialigned $\left(\theta_{1} \geq 150^{\circ}\right) \mathrm{BBH}$ distributions conversely shift to lower values as the predictions are made later in the inspiral. We provide the mean and standard deviation of the final spins predicted for these 6 new sets of partially aligned BBH distributions for all 5 formulas in Table I.
To explore the dependence of these effects on the mass ratio, we have provided histograms of the predicted final spins for these same BBH spin distributions with $q=1 / 3$ in Fig. 9. The discrete peaks at low values of $\chi_{f}$ in the histograms in the top panel are an artifact of the 10 discrete values of $\cos \theta_{1}$ in our $10 \times 10 \times 10$ grid. Each peak contains 100 points with the same initial value of $\theta_{1}$. The decrease in the width of each peak as the BBHs inspiral from $r_{i}=1000 M$ to $r_{f}=10 M$ is a consequence of the antialignment of the $\mathrm{BBH}$ spins for large $\theta_{1}$, but the gaps between the peaks would be filled in if we used a finer grid. The shifts in the mean values of the peaks should be robust with respect to the grid spacing. These shifts for the initially aligned $\mathrm{BBH}$ distributions are provided in Table I for all 5 formulas for $q=1 / 3$, as well as for the intermediate mass ratio $q=2 / 3$.

\section{B. Spin directions}

Before providing quantitative results, we need to clarify what is meant by the direction of the spin of the final black hole. In what reference frame is this direction defined? To avoid the uncertainty associated with a choice of reference

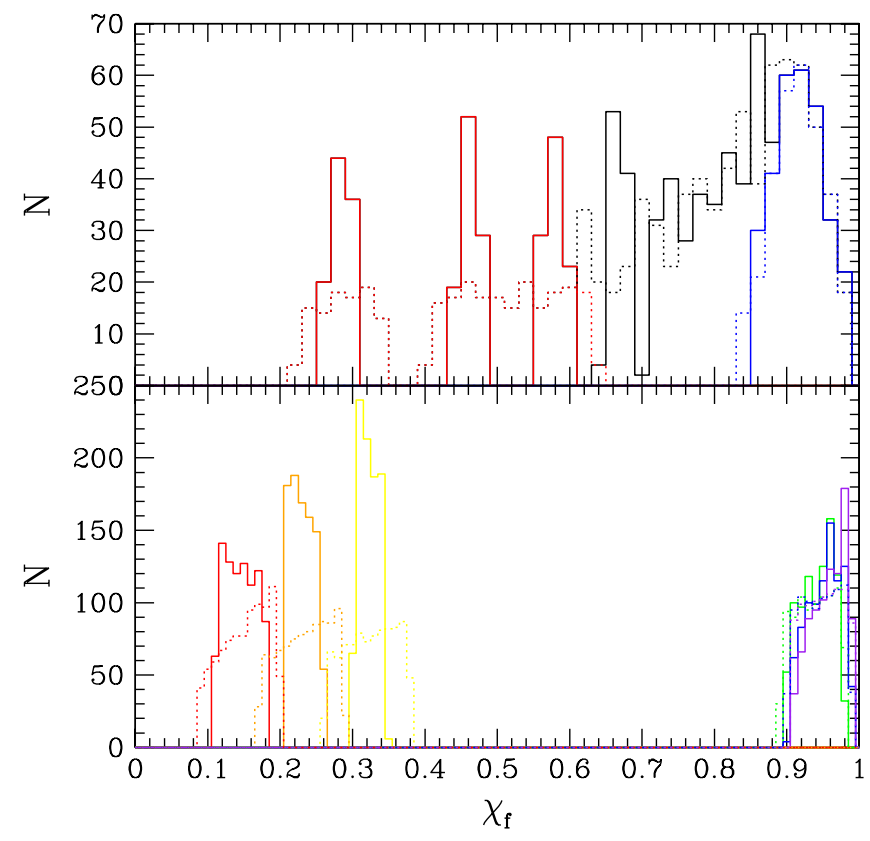

FIG. 9 (color online). Histograms of the final spins $\chi_{f}$ predicted by the AEIn formula for the same sets of BBHs presented in Fig. 8, but with the mass ratio $q=1 / 3$ instead of $q=9 / 11$. As in that figure, the predictions made at $r_{i}=1000 M$ are shown with dotted curves, and those made at $r_{f}=10 M$ are shown with solid curves. The black curves in the top panel show the full set of $1000 \mathrm{BBHs}$, while the blue (red) curves show the subset of $300 \mathrm{BBHs}$ with the lowest (highest) initial values of $\theta_{1}$. In the lower panel, the red, orange, and yellow curves show BBHs with $\chi_{1}$ initially antialigned with $\mathbf{L}_{N}\left(\theta_{1}=170^{\circ}, 160^{\circ}, 150^{\circ}\right)$. The green, blue, and purple curves show BBHs with $\boldsymbol{\chi}_{1}$ initially aligned with $\mathbf{L}_{N}\left(\theta_{1}=30^{\circ}, 20^{\circ}, 10^{\circ}\right)$. 
frame, spin directions are often specified as the angle between the final spin $\boldsymbol{\chi}_{f}$ and a particular direction such as that of the BBH orbital angular momentum $\mathbf{L}_{N}(r)$ at a separation $r$. NR simulations can be used to determine the angle

$$
\vartheta_{f}(r) \equiv \arccos \left[\hat{\mathbf{L}}_{N}(r) \cdot \boldsymbol{\chi}_{f}\right]
$$

between $\mathbf{L}_{N}$ at the separation $r \simeq r_{\mathrm{NR}}$ where the NR simulations begin and the final numerically determined spin $\boldsymbol{\chi}_{f} \simeq \boldsymbol{\chi}_{f}^{\mathrm{NR}}$. Fitting formulas calibrated with NR simulations, such as those proposed in [30,39,48], provide an estimate $\hat{\boldsymbol{\chi}}_{f}^{\text {est }}(r)$ of the final spin based on the BBH spins evaluated at a separation $r$ before merger. Their estimate

$$
\vartheta_{f}^{\text {est }}(r) \equiv \arccos \left[\hat{\mathbf{L}}_{N}(r) \cdot \hat{\boldsymbol{\chi}}_{f}^{\text {est }}(r)\right]
$$

for the angle $\vartheta_{f}$ thus implicitly depends on the separation $r$ both through $\hat{\mathbf{L}}_{N}(r)$ and $\hat{\boldsymbol{\chi}}_{f}^{\text {est }}(r)$. The analytical predictions of BKL and Kes were designed to apply to BBH spin configurations at $r_{\text {ISCO }}$. If $\mathrm{BBH}$ spins are specified at a separation $r \gtrsim r_{\text {ISCO }}$ just a few orbits before merger, the hierarchy of time scales shown in Eq. (3.1) implies that the BKL and Kes estimates $\hat{\boldsymbol{\chi}}_{f}^{\text {est }}$ for the final spin can also be inserted into the right-hand side of Eq. (5.2) to provide estimates $\vartheta_{f}^{\text {est }}$. This angle $\vartheta_{f}(r)$ evaluated at $r \simeq r_{f}$ is physically interesting because it quantifies the alignment between $\boldsymbol{\chi}_{f}$ and $\hat{\mathbf{L}}_{N}$ near merger. GW templates of the late inspiral depend on $\hat{\mathbf{L}}_{N}\left(r_{f}\right)$, while ringdown templates depend on $\boldsymbol{\chi}_{f}$. Estimates of $\vartheta_{f}\left(r_{f}\right)$ might help connect templates from these different stages of the merger.

Astrophysicists might be interested in the alignment between $\boldsymbol{\chi}_{f}$ and the inner edge of the circumbinary disk, which torques might align with $\mathbf{L}_{N}$ at some larger separation $r_{i}$. In that case, we would need to estimate $\vartheta_{f}\left(r_{i}\right)$ at $r_{i} \gg r_{f}$. This angle is more difficult to predict, as the $\mathrm{BBH}$ spins will precess many times during the time $t_{\mathrm{GW}} \gg t_{p}$ it takes the BBHs to inspiral from $r_{i}$ to a separation $r \simeq r_{f}$ where reliable NR simulations begin and the fitting formulas can be trusted. If the BBH spins were specified at $r=r_{i}$, the proper way to predict $\boldsymbol{\chi}_{f}^{\text {est }}\left(r_{i}\right)$ would be to use $\mathrm{PN}$ equations like those in Sec. II to propagate these spins and $\mathbf{L}_{N}$ down to $r_{f}$, and then insert them into the fitting formula of one's choice. Inserting the BBH spins specified at $r_{i}$ directly into a fitting formula, which is essentially equivalent to assuming that they do not precess between $r_{i}$ and $r_{f}$, can lead to large errors in predictions of $\vartheta_{f}$, as shown in [30]. The great advantage of the AEIn formula proposed in [30] is that it claims to be able to predict $\vartheta_{f}\left(r_{i}\right)$ without this PN calculation of spin precession during the inspiral.

The AEIn formula is based on the conjecture that $\boldsymbol{\chi}_{f}$ points in the direction of the total angular momentum $\mathbf{J}$ at any separation, since angular momentum is always radiated parallel to $\mathbf{J}$, thus preserving its direction. This con- jecture is plausible because at large separations, the precession time $t_{p}$ is much shorter than the inspiral time $t_{\mathrm{GW}}$. If the vectors associated with the $\mathrm{BBHs}$ precess rapidly enough, all components except those parallel to $\mathbf{J}$ (which varies on the longer time scale $t_{\mathrm{GW}}$ ) will average to zero. The AEIn conjecture is very useful because it allows $\hat{\boldsymbol{\chi}}_{f}^{\text {est }}(r)$ to be estimated at any separation $r$, even $r_{i} \gg r_{f}$, without solving any PN equations. However, the approximation $t_{p} \ll t_{\mathrm{GW}}$ upon which it depends breaks down at small separations. This may lead to incomplete cancellation of the angular momentum radiated perpendicular to $\mathbf{J}$.

We test this possibility by calculating

$$
\theta_{J} \equiv \arccos \left[\hat{\mathbf{J}}\left(r_{i}\right) \cdot \hat{\mathbf{J}}\left(r_{f}\right)\right],
$$

the angle between the total angular momentum at $r_{i}=$ $1000 M$ and that after the BBHs have inspiraled to $r_{f}=$ $10 M$. If the direction of $\mathbf{J}$ really was preserved during the inspiral, $\theta_{J}$ would vanish. We present histograms of $\theta_{J}$ for mass ratio $q=9 / 11$ in Fig. 10. The upper panel shows the $10 \times 10 \times 10$ grid of $\mathrm{BBH}$ spin configurations evenly spaced in $\left(\cos \theta_{1}, \cos \theta_{2}, \Delta \phi\right)$ that we have discussed previously. The direction of $\mathbf{J}$ changes by $\theta_{J} \lesssim 2^{\circ}$ during most of the inspirals, though a tail extends to larger values for large initial values of $\theta_{1}$. This tail can be seen more

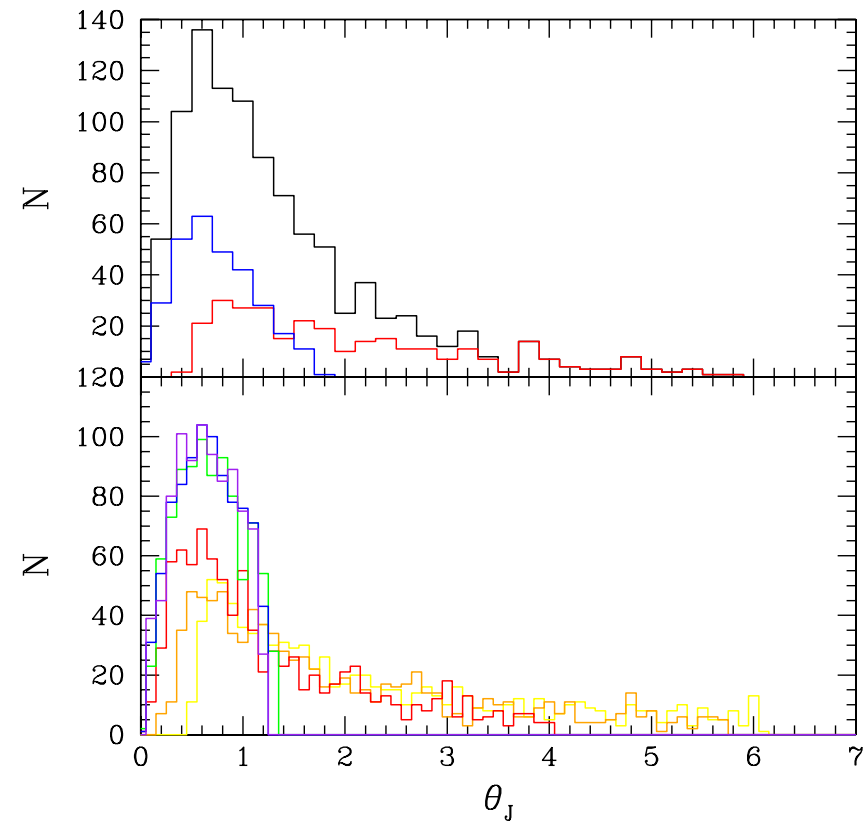

FIG. 10 (color online). Top panel: Histogram of the angle $\theta_{J}$ (in degrees) between the total angular momentum $\mathbf{J}$ at $r_{i}=$ $1000 M$ and that at $r_{f}=10 M$ for our set of 1000 BBHs with $q=$ $9 / 11$ and initially isotropic spins. As in previous figures, the blue (red) curve shows the subset of $300 \mathrm{BBHs}$ with the lowest (highest) initial values of $\theta_{1}$. Bottom panel: Histograms of $\theta_{J}$ for the 6 sets of $900 \mathrm{BBH}$ mergers with flat distributions in $\cos \theta_{2}$ and $\Delta \phi$ at $r_{i}=1000 M$. The red, orange, yellow, green, blue, and purple curves show BBHs that have $\theta_{1}=170^{\circ}, 160^{\circ}, 150^{\circ}$, $30^{\circ}, 20^{\circ}$, and $10^{\circ}$, respectively, at this initial separation. 
clearly in the bottom panel for the BBHs with $\chi_{1}$ initially antialigned with $\mathbf{L}_{N}\left(\theta_{1} \geq 150^{\circ}\right)$. We agree with [30] that these large changes in the direction of $\mathbf{J}$ are likely a consequence of the transitional precession first identified in Ref. [52]. This transitional precession occurs to an even greater extent for smaller mass ratios, as can be seen in Fig. 11 for $q=1 / 3$. As in the upper panel of Fig. 9, discrete peaks resulting from the grid spacing in $\cos \theta_{1}$ can be seen in the left panel of Fig. 11. The middle panel shows that the direction of $\mathbf{J}$ remains nearly constant $\left(\theta_{J} \lesssim\right.$ $\left.0.5^{\circ}\right)$ when $\chi_{1}$ is closely aligned with $\mathbf{L}_{N}\left(\theta_{1} \leq 30^{\circ}\right)$. However, the right panel shows that the assumption of constant $\hat{\mathbf{J}}$ fails badly for the BBHs with $\theta_{1} \geq 150^{\circ}$, which comprise $\sim 7 \%$ of isotropically distributed $\mathrm{BBH}$ mergers. The mass ratio $q=1 / 3$ is not extreme compared to the majority of astrophysical mergers, so caution should be taken when assuming that $\boldsymbol{\chi}_{f}^{\text {est }}\left(r_{i}\right)$ points in the direction of $\mathbf{J}$ in Eq. (5.2).

What about the less ambitious predictions of $\vartheta_{f}(r)$ from BBH spins specified at $r_{f}=10 M$ ? Spin-orbit resonances have significant implications for these predictions as well. We show predictions of $\vartheta_{f}$ by the AEIn formula for a mass ratio of $q=9 / 11$ in Fig. 12. The other formulas predict very similar results. As in Figs. 8 and 9, the dotted curves

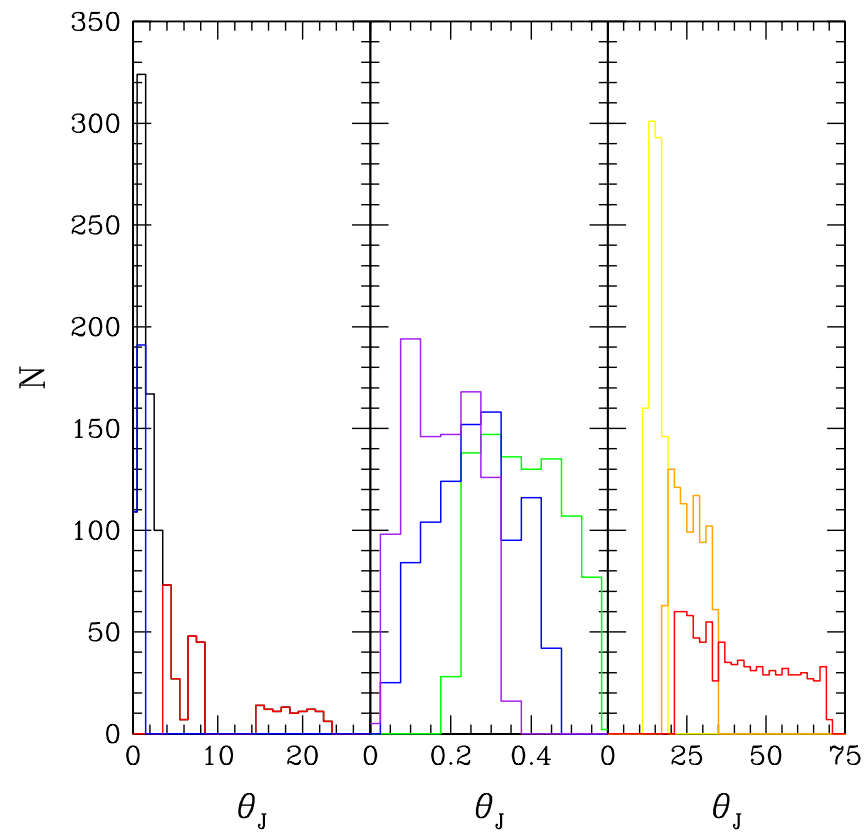

FIG. 11 (color online). Left panel: Histogram of the angle $\theta_{J}$ (in degrees) between the total angular momentum $\mathbf{J}$ at $r_{i}=$ $1000 M$ and that at $r_{f}=10 M$ for our set of 1000 initially isotropically spinning BBHs with $q=1 / 3$. As in previous figures, the blue (red) curve shows the subset of $300 \mathrm{BBHs}$ with the lowest (highest) initial values of $\theta_{1}$. Middle panel: Histograms of $\theta_{J}$ for the 3 sets of $900 \mathrm{BBH}$ mergers initially with $\theta_{1}=10^{\circ}$ (purple), $20^{\circ}$ (blue), and $30^{\circ}$ (green). Right panel: Histograms of $\theta_{J}$ for the 3 sets of $900 \mathrm{BBH}$ mergers initially with $\theta_{1}=150^{\circ}$ (yellow), $160^{\circ}$ (orange), and $170^{\circ}$ (red).

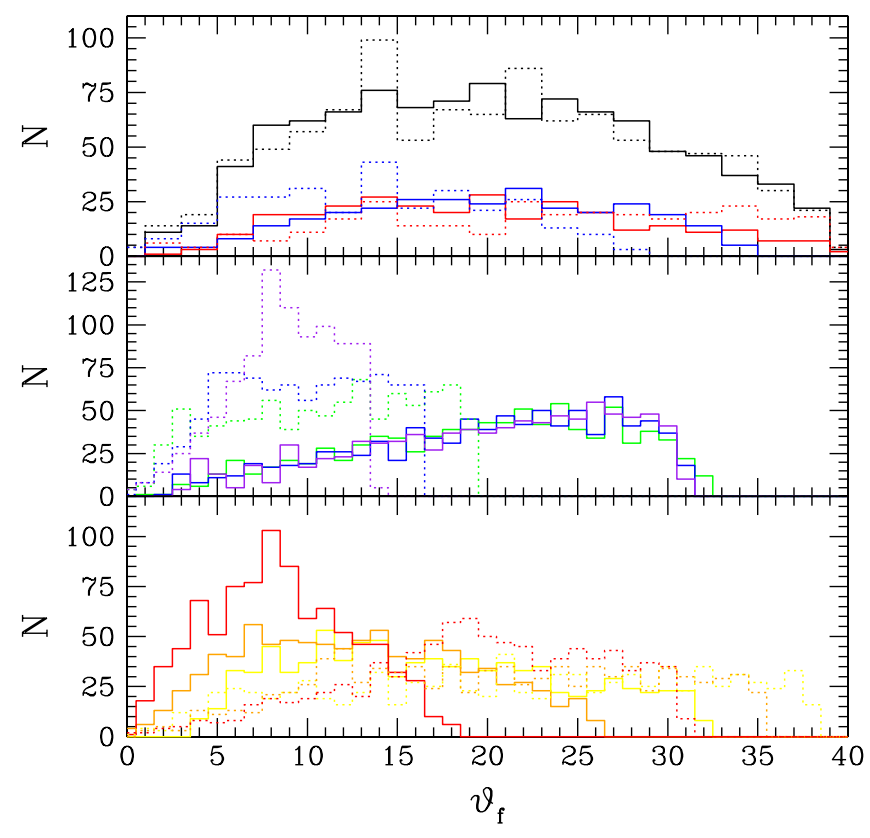

FIG. 12 (color online). Top panel: Histogram of the angle $\vartheta_{f}$ (in degrees) between the orbital angular momentum $\mathbf{L}_{N}$ at $r_{f}=$ $10 M$ and the final spin $\boldsymbol{\chi}_{f}$ predicted by the AEIn formula from the $\mathrm{BBH}$ spins at that separation. The BBHs have a mass ratio $q=9 / 11$. As in previous figures, the black curves show 1000 mergers with initially isotropic $\mathrm{BBH}$ spins, while the blue (red) curves show the subset of $300 \mathrm{BBHs}$ with the lowest (highest) initial values of $\theta_{1}$. The dotted curves show predictions in the absence of spin precession, while the solid curves show how these predictions change when the $\mathrm{BBH}$ spins precess from $r_{i}=$ $1000 M$ to $r_{f}=10 M$ according to the PN equations of Sec. II. Middle panel: Histograms of $\vartheta_{f}$ for the 3 sets of $900 \mathrm{BBH}$ mergers with $\boldsymbol{\chi}_{1}$ initially aligned with $\mathbf{L}_{N}\left[\theta_{1}=10^{\circ}\right.$ (purple), $20^{\circ}$ (blue), $30^{\circ}$ (green)]. Bottom panel: Histograms of $\vartheta_{f}$ for the 3 sets of $900 \mathrm{BBH}$ mergers with $\boldsymbol{\chi}_{1}$ initially antialigned with $\mathbf{L}_{N}$ $\left[\theta_{1}=150^{\circ}\right.$ (yellow), $160^{\circ}$ (orange), $170^{\circ}$ (red)].

show predictions assuming that the initial BBH spin distribution specified at $r_{i}=1000 \mathrm{M}$ is preserved down to $r_{f}=10 M$. The solid curves include spin precession from $r_{i}$ to $r_{f}$ according to the PN equations of Sec. II. The difference between the dotted and solid black curves in the top panel is below the Poisson fluctuations, another consequence of the finding of Refs. [23,26,27] that isotropically oriented $\mathrm{BBH}$ spins remain nearly isotropic as they inspiral. Careful examination of the upper panel reveals that spin precession has shifted the BBHs with $\boldsymbol{\chi}_{1}$ initially aligned with $\mathbf{L}_{N}$ (blue distribution) to larger $\vartheta_{f}$, while the antialigned BBHs have conversely shifted to smaller $\vartheta_{f}$.

This trend is much more pronounced in the middle and bottom panels of Fig. 12. Spin precession actually results in the initially aligned BBHs $\left(\theta_{1} \leq 30^{\circ}\right)$ having larger values of $\vartheta_{f}^{\text {est }}(r)$ at $r_{f}=10 M$ than the antialigned BBHs $\left(\theta_{1} \geq\right.$ $\left.150^{\circ}\right)$, a reversal of what would have been predicted if $\vartheta_{f}^{\text {est }}(r)$ was calculated from the spin distributions at $r_{i}$ 


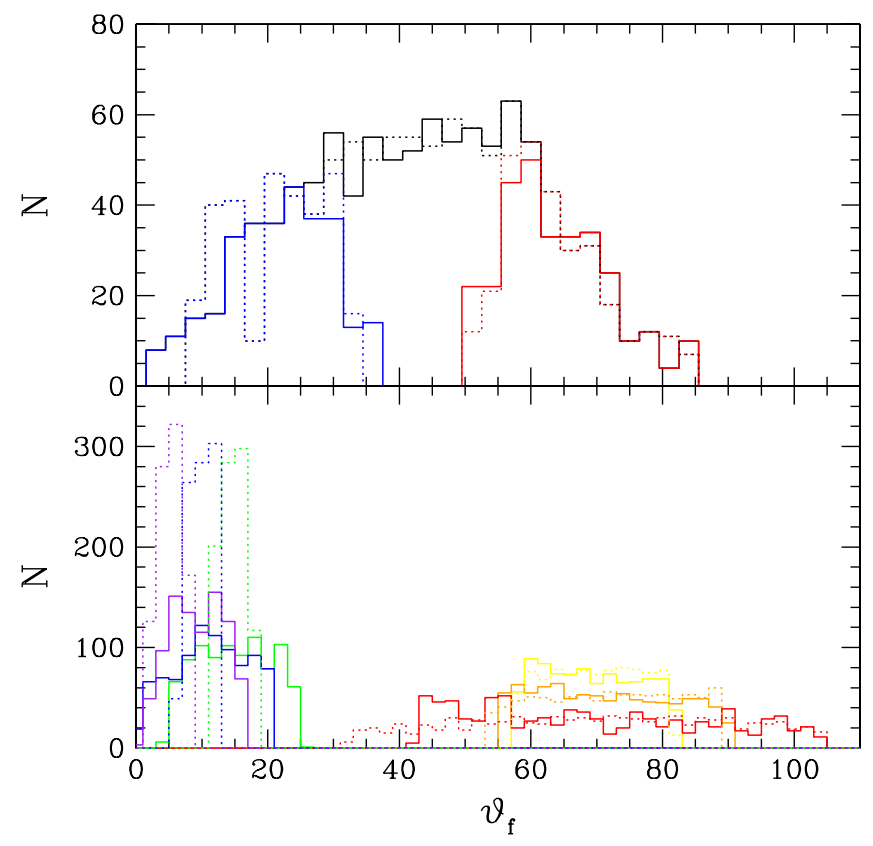

FIG. 13 (color online). Histograms of the angle $\vartheta_{f}$ predicted by the AEIn formula for the same sets of BBHs shown in Fig. 12 but with a mass ratio $q=1 / 3$. As in that figure, the top panel shows BBHs with initially isotropic spins with the blue (red) curves indicating those BBHs with the lowest (highest) initial values of $\theta_{1}$. Dotted curves show predictions without spin precession, while the solid curves show how these predictions change if the BBHs spins precess from $r_{i}=1000 M$ to $r_{f}=$ $10 M$ according to the PN equations of Sec. II. The bottom panel shows distributions with flat initial distributions of $\cos \theta_{2}$ and $\Delta \phi$, but with $\theta_{1}$ now initially set to $170^{\circ}, 160^{\circ}, 150^{\circ}, 30^{\circ}, 20^{\circ}$, and $10^{\circ}$, respectively, for the red, orange, yellow, green, blue, and purple curves.

without spin precession as shown by the dotted curves. The spin-orbit resonances explain this highly counterintuitive result. The BBHs initially with $\theta_{1} \leq 30^{\circ}$ are influenced by the $\Delta \phi=0^{\circ}$ resonances that align the $\mathrm{BBH}$ spins with each other and antialign $\mathbf{S}=\mathbf{S}_{1}+\mathbf{S}_{2}$ with $\mathbf{L}_{N}$. Both effects lead to larger predicted values of $\vartheta_{f}$. Conversely, the BBHs initially with $\theta_{1} \geq 150^{\circ}$ are influenced by the $\Delta \phi=180^{\circ}$ resonances, which greatly decrease the magnitude of $\mathbf{S}$ and align it with $\mathbf{L}_{N}$. This explains the reduced values of $\vartheta_{f}$ for these BBHs seen in the bottom panel of Fig. 12. This same effect can be seen for a mass ratio of $q=1 / 3$ in Fig. 13, albeit with less significance owing to the weaker spin alignment at this smaller mass ratio. Figures 12 and 13 again illustrate the importance of accounting for spin precession between $r_{i}=1000 M$ and $r_{f}=10 M$ when attempting to predict final spins.

\section{SPIN-PRECESSION UNCERTAINTY}

We focused so far on how spin precession between $r_{i}$ and $r_{f}$ alters the expected distribution of final spins. In this section, we show that spin precession introduces a funda- mental uncertainty in predicting the final spin for a particular merger. An uncertainty $\Delta r$ in the BBH separation leads to an uncertainty $\Delta t_{\mathrm{GW}}$ in the time until merger. If this uncertainty is comparable to the precession time $t_{p}$, the phase of the spin precession at which the merger occurs will be uncertain as well. This new uncertainty is independent of and may exceed that associated with the NR simulations themselves. Readers only interested in astrophysical distributions of final spins may wish to proceed to the discussion in Sec. VII.

To illustrate the origin of this uncertainty, we show a few characteristic examples of how the predicted spin magnitude and direction evolve during the PN inspiral. In Fig. 14 we display the final spin magnitude $\chi_{f}^{\text {est }}(r)$ and the angle $\vartheta_{f}^{\text {est }}(r)$ as predicted by the AEIn and the Kesden formulas for a binary with mass ratio $q=9 / 11$, extremal spins, and initial spin orientation specified by the angles $\theta_{1}=120^{\circ}$, $\theta_{2}=60^{\circ}$, and $\Delta \phi=288^{\circ}$. The behavior of the AEIo, FAU, and BKL formulas is quite similar to the Kesden formula. The different curves in each panel correspond to slightly different initial frequencies or separations, $M \omega_{i}=$ $3.16 \times 10^{-5}, 3.17 \times 10^{-5}, 3.18 \times 10^{-5}$, and $3.19 \times 10^{-5}$. The spin precession manifests itself in the oscillatory character of the curves; these oscillations would be absent for the resonant configurations described in Sec. III that evolve on the longer time scale $t_{\mathrm{GW}}$. The thin solid lines represent envelope functions obtained by fitting fourthorder polynomials to the maxima and minima, respectively, of the evolutions starting with $M \omega_{i}=3.16 \times$ $10^{-5}$. Note that these fits contain no information on the results obtained by using different values of $M \omega_{i}$, and yet they still provide excellent envelopes in all cases.

This figure illustrates two ambiguities in predicting $\boldsymbol{\chi}_{f}$ : (i) the initial frequency $\omega_{i}$ at which the $\mathrm{BBH}$ parameters are specified, and (ii) the final separation $r_{f}$ at which the given formula for $\boldsymbol{\chi}_{f}$ should be applied. Uncertainty in the separation at which the binary decouples from external interactions could lead to ambiguity in $\omega_{i}$ in theoretical studies, while uncertainty in the observed distance, projected separation, or line-of-sight velocity could lead to uncertainty in $\omega_{i}$ for models of particular systems. Differing gauge choices or definitions of $r_{f}$ in PN calculations and NR simulations could lead to uncertainty in the separation at which fitting formulas should be applied. Our task in evaluating the resulting uncertainties for the fitting formulas AEIn, AEIo, FAU, BKL, and Kes introduced in Sec. V is somewhat simplified because both ambiguities are rooted in the rapid variations of the phase and in the resulting oscillations in the final quantities. These precession-induced oscillations are a clear manifestation of the hierarchy of time scales introduced in Eq. (3.1): $t_{p} \ll t_{\mathrm{GW}}$.

In the remainder of this section, we discuss the uncertainties in the final spin magnitude $\chi_{f}^{\text {est }}(r)$ and the angle $\vartheta_{f}^{\text {est }}(r)$ caused by the rapid spin precession. These quanti- 

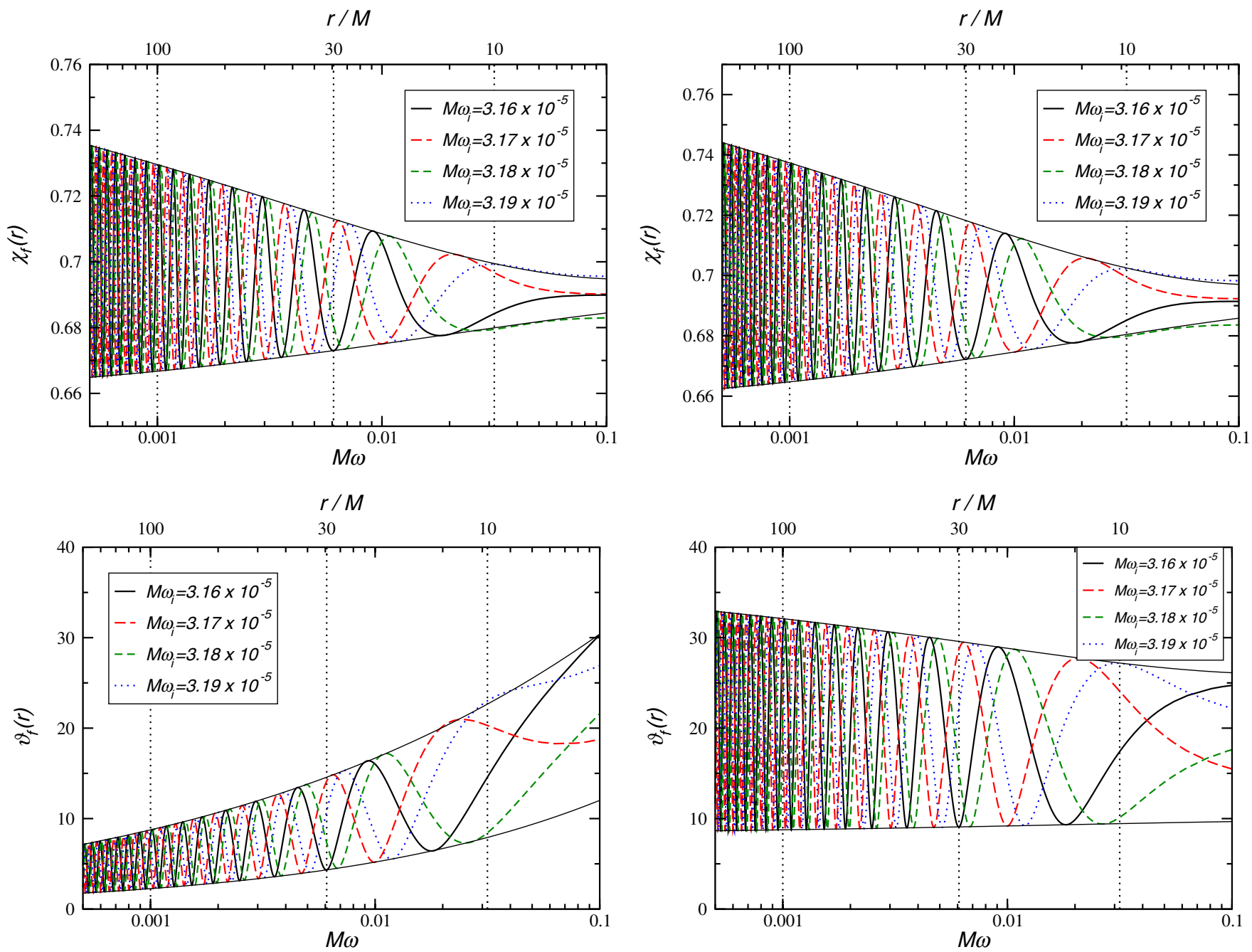

FIG. 14 (color online). Predicted $\chi_{f}$ (upper panels) and $\vartheta_{f}(r)$ (lower panels) obtained from the AEIn (left) and the Kesden (right) formula. The initial parameters of the binary are $q=9 / 11, \chi_{1}=\chi_{2}=1, \theta_{1}=120^{\circ}, \theta_{2}=60^{\circ}$, and $\Delta \phi=288^{\circ}$. The different curves correspond to initial frequencies $M \omega_{i}=3.16 \times 10^{-5}$ (solid line), $3.17 \times 10^{-5}$ (long-dashed line), $3.18 \times 10^{-5}$ (dashed line), and $3.19 \times 10^{-5}$ (dotted line). The envelope determined for $M \omega_{i}=3.16 \times 10^{-5}$ is displayed by thin solid curves. The upper horizontal axis gives the binary separation in units of $M$; the lower horizontal axis gives the corresponding orbital frequency $M \omega$.

ties are important for modeling the assembly of supermassive black holes in the context of cosmological structure formation (see e.g. [24,53-57]). They are also relevant for electromagnetic counterparts of gravitational-wave sources [58], especially when the invoked mechanism producing the counterparts depends on the recoil velocity of the remnant black hole [59-61].

We determine the precession-induced uncertainties as follows. Individual evolutions, such as those considered in Fig. 14, suggest that the width of the envelopes is an excellent estimate for how $\chi_{f}^{\text {est }}(r)$ and $\vartheta_{f}^{\text {est }}(r)$ change with variations in $\omega_{i}$ [ambiguity (i) above] or the separation $r$ [ambiguity (ii)]. We have verified this conjecture by evolving the evenly spaced $10 \times 10 \times 10$ grid of initially isotropic, maximally spinning $\mathrm{BBH}$ configurations introduced in Sec. IV for mass ratio $q=2 / 3$ and several slightly different initial frequencies. When we estimate uncertainties by varying $M \omega_{i}$ from $3.16 \times 10^{-5}$ to $3.22 \times$ $10^{-5}$ in steps of $0.005 \times 10^{-5}$, we obtain the red dashed histograms in Fig. 15. These histograms are in good agreement with the black solid histograms, where the uncertainty was estimated from the width of the envelopes. In order to reduce computational cost, in the remainder of this section we determine the uncertainties $\Delta \chi_{f}(r)$ and $\Delta \vartheta_{f}(r)$ by evolving an ensemble of binaries from a single initial frequency $\left(M \omega_{i}=3.16 \times 10^{-5}\right)$ and using the envelope method.

We have evolved the uniform $10 \times 10 \times 10$ grid of maximally spinning binaries introduced in Sec. IV for three different mass ratios: $q=9 / 11, q=2 / 3$, and $q=$ $1 / 3$. The average uncertainties (plus or minus their associated standard deviations) are summarized in Table II.

Errors in the final spin magnitudes due to the rapid spin precession are in the range $\Delta \chi_{f} \lesssim 0.03$ for all mass ratios. 

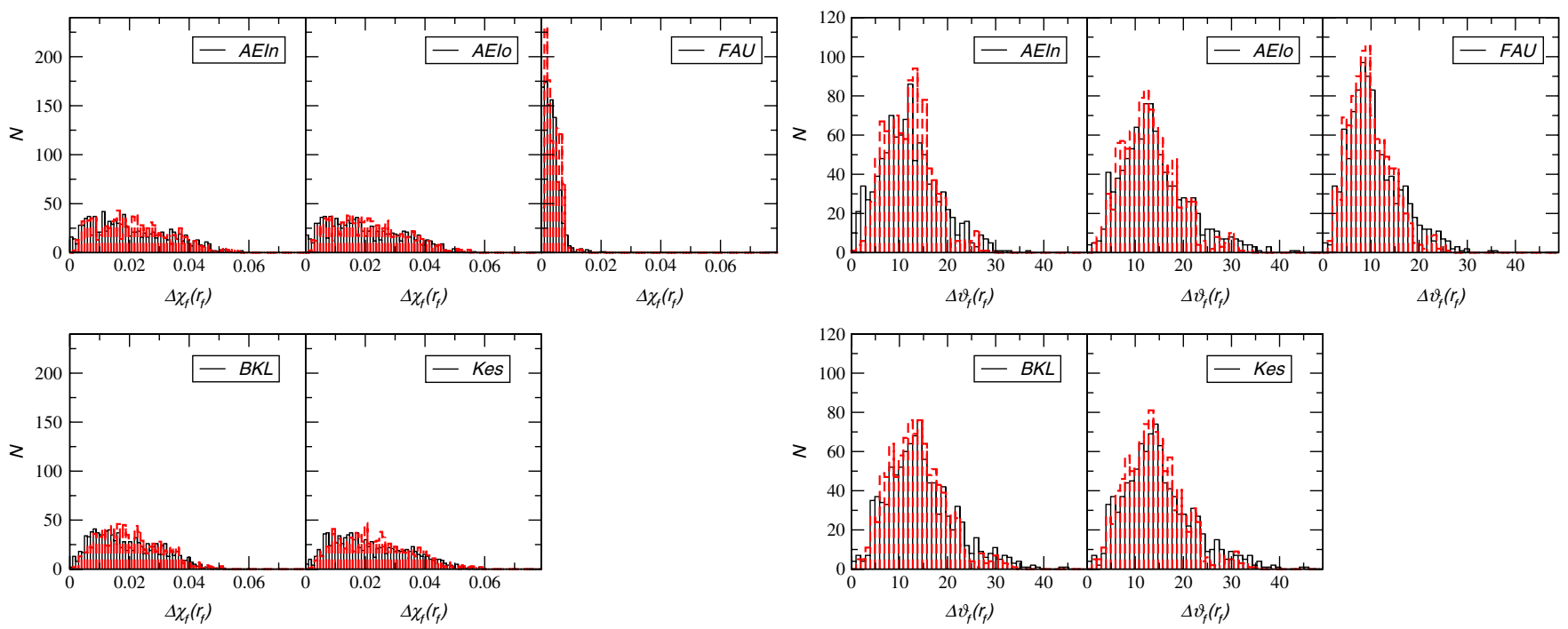

FIG. 15 (color online). Uncertainties in the final spin magnitude $\chi_{f}\left(r_{f}\right)$ (left) and direction $\vartheta_{f}\left(r_{f}\right)$ (right) for extremal BBHs with mass ratio $q=2 / 3$. Solid black histograms were obtained by starting the evolutions at $M \omega_{i}=3.16 \times 10^{-5}$ and using the envelope method of Fig. 14. Dashed red histograms were obtained by considering the maximum variation in the final quantities as we let $M \omega_{i}$ vary from $3.16 \times 10^{-5}$ to $3.22 \times 10^{-5}$ in steps of $0.005 \times 10^{-5}$.

The FAU formula performs exceptionally well for nearly equal masses, although it deteriorates to the level of the other predictions for $q=1 / 3$. We suspect that this is because several of the higher-order terms in $\eta$ in the FAU formula are symmetric in the dimensionless spins $\boldsymbol{\chi}_{1}, \boldsymbol{\chi}_{2}$, while physically one would expect the spin of the more massive black hole to be more important in the limit $q \rightarrow 0$. Overall, however, all formulas are able to predict the spin magnitude with rather good accuracy.

TABLE II. Uncertainty distributions in $\chi_{f}$ and in the various angles describing the final spin directions, as predicted by the formulas listed in Sec. V. The uncertainties and their standard deviations are obtained by evolving uniform $10 \times 10 \times 10$ grids of maximally spinning BBHs with mass ratio $q=9 / 11,2 / 3$, and $1 / 3$, respectively.

\begin{tabular}{lcccc}
\hline \hline Model & $q$ & $\Delta \chi_{f}(r=10 M)$ & $\Delta \vartheta_{f}(r=10 M)$ & $\Delta \vartheta_{f}(r=1000 M)$ \\
\hline AEIn & $9 / 11$ & $0.0159 \pm 0.0099$ & $8.38 \pm 5.30$ & $1.47 \pm 1.09$ \\
AEIo & $9 / 11$ & $0.0155 \pm 0.0098$ & $11.38 \pm 6.18$ & $6.55 \pm 2.73$ \\
FAU & $9 / 11$ & $0.0021 \pm 0.0035$ & $8.51 \pm 4.75$ & $3.67 \pm 1.68$ \\
BKL & $9 / 11$ & $0.0153 \pm 0.0094$ & $11.74 \pm 6.39$ & $6.89 \pm 2.92$ \\
Kes & $9 / 11$ & $0.0174 \pm 0.0105$ & $11.99 \pm 6.51$ & $7.04 \pm 2.96$ \\
AEIn & $2 / 3$ & $0.0205 \pm 0.0127$ & $11.96 \pm 6.17$ & $1.81 \pm 1.21$ \\
AEIo & $2 / 3$ & $0.0199 \pm 0.0124$ & $14.10 \pm 6.99$ & $7.37 \pm 2.80$ \\
FAU & $2 / 3$ & $0.0034 \pm 0.0026$ & $10.66 \pm 5.61$ & $4.41 \pm 1.76$ \\
BKL & $2 / 3$ & $0.0191 \pm 0.0108$ & $14.52 \pm 7.05$ & $7.79 \pm 2.98$ \\
Kes & $2 / 3$ & $0.0217 \pm 0.0124$ & $14.83 \pm 7.24$ & $8.02 \pm 2.99$ \\
AEIn & $1 / 3$ & $0.0165 \pm 0.0109$ & $8.58 \pm 4.17$ & $3.96 \pm 4.46$ \\
AEIo & $1 / 3$ & $0.0156 \pm 0.0101$ & $9.57 \pm 4.62$ & $10.45 \pm 4.12$ \\
FAU & $1 / 3$ & $0.0177 \pm 0.0090$ & $7.80 \pm 3.84$ & $6.60 \pm 2.75$ \\
BKL & $1 / 3$ & $0.0148 \pm 0.0089$ & $9.81 \pm 4.79$ & $11.24 \pm 4.45$ \\
Kes & $1 / 3$ & $0.0167 \pm 0.0105$ & $10.01 \pm 5.06$ & $11.49 \pm 4.48$ \\
\hline \hline
\end{tabular}

The uncertainty $\Delta \vartheta_{f}\left(r_{f}\right)$ in the angle between the final spin and the orbital angular momentum shortly before merger is typically in the range of a few to $20^{\circ}$. Investigation of the angular dependence of the spin uncertainties shows that the AEIn formula tends to behave better for initially aligned spins (small $\theta_{1}$ and $\theta_{2}$ ) and worse for antialigned cases. This is likely a consequence of antialigned binaries being closer to the limit $\mathbf{L}(r) \approx-\mathbf{S}(r)$ where transitional precession [52] occurs, violating assumptions (iii) and (iv) of Ref. [30].

All formulas are able to predict the angle $\vartheta_{f}\left(r_{i}\right)$ between the orbital angular momentum $\mathbf{L}_{N}\left(r_{i}\right)$ and the final spin with decent accuracy if the BBH spins and $\mathbf{L}_{N}$ are evolved inwards to $r_{f}$ according to the PN equations of Sec. II. The AEIn predictions are more accurate overall, but investigation of the angular dependence reveals that this accuracy deteriorates (as expected) when $q=1 / 3$ and the spin of the larger black hole is nearly antialigned. In this limit the uncertainties increase up to $\sim 20^{\circ}$. This is again a consequence of those configurations approaching the transitional precession regime, where $\mathbf{L}(r) \approx-\mathbf{S}(r)$.

The AEIn prediction is unique in that it claims to predict $\vartheta_{f}\left(r_{i}\right)$ using the binary parameters at large separation without PN evolution. Our findings confirm (quite remarkably) that the majority of binaries in an initially isotropic ensemble result in a final spin that is nearly aligned with the orbital angular momentum at large binary separation. The values of $\theta_{J}$ shown in Figs. 10 and 11 suggest that this would not be the case for BBHs initially antialigned with $\mathbf{L}_{N}$. The accuracy of the AEIn predictions also decreases for unequal masses (as expected and verified by our results for $q=1 / 3$ ). More extreme mass ratios are expected to play a significant and possibly dominant role in the coales- 
cence of SBH binaries [62-64], so it will be crucial to test the robustness of the AEIn predictions for $q=1 / 10$ and beyond. Accurate PN evolutions are more difficult in this regime, and we plan to investigate more extreme mass ratios in the future.

\section{DISCUSSION}

In this paper, we examined how precession affects the distribution of spin orientations as BBHs inspiral from initial separations $r_{i} \approx 1000 M$ where gravitational radiation begins to dominate the dynamics, all the way down to separations $r_{f} \simeq 10 M$ where numerical-relativity simulations typically begin.

We confirmed previous findings that isotropic spin distributions at $r_{i} \simeq 1000 M$ remain isotropic at $r_{f} \simeq 10 M$ $[23,26,27]$. However, torques exerted by circumbinary disks may partially align BBH spins with the orbital angular momentum at separations $r>r_{i}$ before gravitational radiation drives the inspiral [23]. Recent simulations suggest that the residual misalignment of the $\mathrm{BBH}$ spins with their accretion disk could typically be $\sim 10^{\circ}\left(30^{\circ}\right)$ for cold (hot) accretion disks, respectively [17]. Partially motivated by these findings, we carried out a more careful analysis of spin distributions that are partially aligned with the orbital angular momentum at $r=r_{i}$. We found that spin precession efficiently aligns the BBH spins with each other when the spin of the more massive black hole is initially partially aligned with the orbital angular momentum, increasing the final spin. We found the opposite trend when the spin of the more massive black hole is initially antialigned with the orbital angular momentum. Long evolutions are necessary to capture the full magnitude of the spin alignment. This could explain why these trends were not observed in the PN evolutions by Lousto et al. [27], which began at a fiducial binary separation $r=50 \mathrm{M}$.

Some models of BBH evolution (see e.g. [62,63]) suggest that SBH mergers might have comparable mass ratios $(q \lesssim 1)$ at high redshift and more extreme mass ratios at low redshift. Since spin alignment is stronger for comparable-mass binaries, more alignment might be expected in SBH binaries at high redshifts. Observational arguments (see e.g. [65]) and magnetohydrodynamic simulations of accretion disks [66] provide some evidence that black hole spins are related to the radio loudness of quasars. If so, the inefficient alignment (and consequently smaller spins) produced by unequal-mass mergers at low redshift would at least be consistent with recent observational claims that the mean radiative efficiency of quasars decreases at low redshift $[67,68]$. Stellar-mass black hole binaries should also have comparable mass ratios, so significant spin alignment could occur in such systems as well.

We also pointed out that predictions $\boldsymbol{\chi}_{f}^{\text {est }}\left(r_{i}\right)$ of the final spin usually suffer from two sources of uncertainty: (i) the uncertainty in the initial separation $r_{i}$ at which the $\mathrm{BBH}$ parameters are specified, and (ii) the uncertainty in the final separation $r_{f}$ to which these parameters should be evolved before applying the given fitting formula. Both ambiguities are rooted in the rapid precessional modulation of the orbital parameters, which in turn results from the precessional time scale $t_{p}$ being much shorter than the radiation time scale $t_{\mathrm{GW}}$. Spin precession induces an intrinsic inaccuracy $\Delta \chi_{f} \lesssim 0.03$ in the dimensionless spin magnitude and $\Delta \vartheta_{f} \lesssim 20^{\circ}$ in the final spin direction.

The spin-orbit resonances studied in this paper significantly affect the distribution of gravitational recoil velocities resulting from $\mathrm{BBH}$ mergers, because the maximum recoil velocity has a strong dependence on spin alignment [15-17]. We investigate the predictions of different formulas for the recoil velocities that have been proposed in the literature in Ref. [41].

\section{ACKNOWLEDGMENTS}

We are particularly grateful to Vitor Cardoso for helping to test our numerical implementation of the PN evolution equations described in Sec. II, and to Étienne Racine for pointing out the possible relevance of the quadrupolemonopole interaction. We would also like to thank Enrico Barausse, Manuela Campanelli, Yanbei Chen, Pablo Laguna, Carlos Lousto, Samaya Nissanke, Evan Ochsner, Sterl Phinney, Luciano Rezzolla, and Manuel Tiglio for useful discussions. This work was supported by grants from the Sherman Fairchild Foundation to Caltech, by NSF Grants No. PHY-0601459 (PI: Thorne) and No. PHY-090003 (TeraGrid), and by FCT-Portugal through Projects No. PTDC/CTE-AST/098034/2008 and No. PTDC/FIS/098032/2008. M. K. acknowledges support from NASA BEFS Grant No. NNX07AH06G (PI: Phinney). E. B.'s research was supported by NSF Grant No. PHY-0900735. U. S. acknowledges support from NSF Grant No. PHY-0652995.
[1] B. F. Schutz, Nature (London) 323, 310 (1986).

[2] D. E. Holz and S. A. Hughes, Astrophys. J. 629, 15 (2005).

[3] M. C. Begelman, R. D. Blandford, and M. J. Rees, Nature (London) 287, 307 (1980).
[4] J. Frank and M. J. Rees, Mon. Not. R. Astron. Soc. 176, 633 (1976).

[5] M. Milosavljevic and D. Merritt, Astrophys. J. 563, 34 (2001). 
[6] D. N. C. Lin and J. Papaloizou, Mon. Not. R. Astron. Soc. 188, 191 (1979).

[7] P. Goldreich and S. Tremaine, Astrophys. J. 241, 425 (1980).

[8] P. Chang, Astrophys. J. 684, 236 (2008).

[9] P. C. Peters, Phys. Rev. 136, B1224 (1964).

[10] A. Buonanno, Y. Chen, and T. Damour, Phys. Rev. D 74, 104005 (2006).

[11] M. Campanelli, C. O. Lousto, H. Nakano, and Y. Zlochower, Phys. Rev. D 79, 084010 (2009).

[12] F. Pretorius, Phys. Rev. Lett. 95, 121101 (2005).

[13] M. Campanelli, C. O. Lousto, P. Marronetti, and Y. Zlochower, Phys. Rev. Lett. 96, 111101 (2006).

[14] J. G. Baker, J. Centrella, D.-I. Choi, M. Koppitz, and J. van Meter, Phys. Rev. Lett. 96, 111102 (2006).

[15] J.A. Gonzalez, M.D. Hannam, U. Sperhake, B. Bruegmann, and S. Husa, Phys. Rev. Lett. 98, 231101 (2007).

[16] M. Campanelli, C. O. Lousto, Y. Zlochower, and D. Merritt, Astrophys. J. 659, L5 (2007).

[17] M. Dotti et al., arXiv:0910.5729.

[18] D. Merritt, M. Milosavljevic, M. Favata, S. A. Hughes, and D. E. Holz, Astrophys. J. 607, L9 (2004).

[19] J. Magorrian et al., Astron. J. 115, 2285 (1998).

[20] L. Ferrarese and D. Merritt, Astrophys. J. 539, L9 (2000).

[21] S. Tremaine et al., Astrophys. J. 574, 740 (2002).

[22] J. M. Bardeen and J. A. Petterson, Astrophys. J. 195, L65 (1975).

[23] T. Bogdanović, C.S. Reynolds, and M.C. Miller, Astrophys. J. 661, L147 (2007).

[24] E. Berti and M. Volonteri, Astrophys. J. 684, 822 (2008).

[25] J.D. Schnittman, Phys. Rev. D 70, 124020 (2004).

[26] F. Herrmann, J. Silberholz, M. Bellone, G. Guerberoff, and M. Tiglio, Classical Quantum Gravity 27, 032001 (2010).

[27] C.O. Lousto, H. Nakano, Y. Zlochower, and M. Campanelli, arXiv:0910.3197 [Phys. Rev. D (to be published)].

[28] L. E. Kidder, Phys. Rev. D 52, 821 (1995).

[29] A. Buonanno, Y.-b. Chen, and M. Vallisneri, Phys. Rev. D 67, 104025 (2003).

[30] E. Barausse and L. Rezzolla, Astrophys. J. Lett. 704, L40 (2009).

[31] E. Barausse, E. Racine, and A. Buonanno, Phys. Rev. D 80, 104025 (2009).

[32] E. Barausse and A. Buonanno, arXiv:0912.3517 [Phys. Rev. D (to be published)]

[33] E. Racine, Phys. Rev. D 78, 044021 (2008).

[34] E. Poisson, Phys. Rev. D 57, 5287 (1998).

[35] B. Mikoczi, M. Vasuth, and L. A. Gergely, Phys. Rev. D 71, 124043 (2005).

[36] E. Racine, A. Buonanno, and L. E. Kidder, Phys. Rev. D 80, 044010 (2009).
[37] W.H. Press, S. A. Teukolsky, W. T. Vetterling, and B.P. Flannery, Numerical Recipes in C++-The Art of Scientific Computing-Third Edition (Cambridge University Press, Cambridge, U.K., 2007), ISBN 0-521-75033-4.

[38] L. Boyle, M. Kesden, and S. Nissanke, Phys. Rev. Lett. 100, 151101 (2008).

[39] L. Boyle and M. Kesden, Phys. Rev. D 78, 024017 (2008).

[40] T. Damour, Phys. Rev. D 64, 124013 (2001).

[41] M. Kesden, U. Sperhake, and E. Berti, arXiv:1003.4993 [Astrophys. J. (to be published)].

[42] A. Buonanno, G. B. Cook, and F. Pretorius, Phys. Rev. D 75, 124018 (2007).

[43] E. Berti et al., Phys. Rev. D 76, 064034 (2007).

[44] A. Buonanno et al., Phys. Rev. D 76, 104049 (2007).

[45] L. Rezzolla et al., Astrophys. J. 679, 1422 (2008).

[46] L. Rezzolla et al., Astrophys. J. 674, L29 (2008).

[47] L. Rezzolla et al., Phys. Rev. D 78, 044002 (2008).

[48] W. Tichy and P. Marronetti, Phys. Rev. D 78, 081501 (2008).

[49] C. O. Lousto, M. Campanelli, and Y. Zlochower, arXiv:0904.3541.

[50] A. Buonanno, L. E. Kidder, and L. Lehner, Phys. Rev. D 77, 026004 (2008).

[51] M. Kesden, Phys. Rev. D 78, 084030 (2008).

[52] T. A. Apostolatos, C. Cutler, G. J. Sussman, and K. S. Thorne, Phys. Rev. D 49, 6274 (1994).

[53] M. Volonteri, F. Haardt, and P. Madau, Astrophys. J. 582, 559 (2003).

[54] T. Tanaka and Z. Haiman, Astrophys. J. 696, 1798 (2009).

[55] C.d.P. Lagos, N.D. Padilla, and S.A. Cora, arXiv:0901.0547.

[56] D. Sijacki, V. Springel, and M. G. Haehnelt, arXiv:0905.1689.

[57] N. Fanidakis et al., arXiv:0911.1128.

[58] M. Dotti, R. Salvaterra, A. Sesana, M. Colpi, and F. Haardt, Mon. Not. R. Astron. Soc. 372, 869 (2006),

[59] Z. Lippai, Z. Frei, and Z. Haiman, arXiv:0801.0739.

[60] J. D. Schnittman and J. H. Krolik, arXiv:0802.3556.

[61] G. A. Shields and E. W. Bonning, arXiv:0802.3873.

[62] S. M. Koushiappas and A. R. Zentner, Astrophys. J. 639, 7 (2006).

[63] A. Sesana, M. Volonteri, and F. Haardt, Mon. Not. R. Astron. Soc. 377, 1711 (2007).

[64] L. A. Gergely and P. L. Biermann, Astrophys. J. 697, 1621 (2009).

[65] M. Volonteri, M. Sikora, and J.-P. Lasota, arXiv:0706.3900.

[66] A. Tchekhovskoy, R. Narayan, and J.C. McKinney, Astrophys. J. 711, 50 (2010).

[67] J. M. Wang et al., Astrophys. J. 697, L141 (2009).

[68] Y.-R. Li, J.-M. Wang, Y.-F. Yuan, and S. Zhang, Astrophys. J. 710, 878 (2010). 